THE AstrophysiCAL JoURNAL, 479:886-901, 1997 April 20

(C) 1997. The American Astronomical Society. All rights reserved. Printed in U.S.A.

\title{
ON THE ROTATION AND MAGNETIC FIELD EVOLUTION OF SUPERCONDUCTING STRANGE STARS
}

\author{
H. F. Chau ${ }^{1,2}$ \\ School of Natural Sciences, Institute for Advanced Study, Olden Lane, Princeton, NJ 08540 \\ Received 1996 February 26; accepted 1996 June 17
}

\begin{abstract}
Are pulsars made up of strange matter? The magnetic field decay of a pulsar may be able to give us an answer. Since Cooper pairing of quarks occurs inside a sufficiently cold strange star, the strange stellar core is superconducting. In order to compensate for the effect of rotation, different superconducting species inside a rotating strange star try to set up different values of London fields. Thus we have a frustrated system. Using Ginzburg-Landau formalism, I solved the problem of a rotating superconducting strange star: Instead of setting up a global London field, vortex bundles carrying localized magnetic fields are formed. Moreover, the number density of vortex bundles is directly proportional to the angular speed of the star. Since it is energetically favorable for the vortex bundles to pin to magnetic flux tubes, the rotational dynamics and magnetic evolution of a strange star are coupled together, leading to magnetic flux expulsion as the star slows down. I investigate this effect numerically and find that the characteristic field decay time is much less than $20 \mathrm{Myr}$ in all reasonable parameter regions. On the other hand, the characteristic magnetic field decay time for pulsars is $\geq 20$ Myr. Thus, my finding casts doubts on the hypothesis that pulsars are strange stars.
\end{abstract}

Subject headings: dense matter - magnetic fields — stars: interiors — stars: magnetic fields stars: rotation

\section{INTRODUCTION}

What is the most stable form of baryonic matter at high density? This question is of both observational and theoretical interest. With the discovery of pulsars and their identification with the neutron stars in the late 1960s, many people thought that neutron star matter is the most stable form of cold condensed matter at high density. This belief was later challenged by Witten. He proposed that, at sufficiently high density, deconfinement of nucleons occurs. Moreover, some of the $d$ and $u$ quarks on the Fermi surface are converted to strange quarks via the weak interaction. Thus, the most stable form of matter at ultrahigh density is a degenerate mixture of $u, d$, and $s$ quarks together with a small amount of electrons so as to maintain overall charge neutrality (Witten 1984). This kind of material state is called strange matter. His idea was examined in detail later by Farhi \& Jaffe (1984).

The possibility of self-gravitating strange matter, namely, a strange star, has also been investigated. The equation of state (EOS) calculations of strange stars suggest that they are indeed stable objects in a certain parameter range (Alcock, Farhi, \& Olinto 1986; Haensel, Zdunik, \& Schaeffer 1986; Benvenuto \& Horvath 1989; see also Madsen \& Haensel 1991 and Benvenuto, Horvath, \& Vucetich 1991b). The mass and radius of a stable strange star are found to be similar to those of a neutron star. Consequently, some authors suggested that pulsars are in fact strange stars (see, e.g., Benvenuto et al. 1991b). In principle, the question of whether pulsars are neutron or strange stars can be answered by comparing the core density of a neutron star to the strange matter transition density. Unfortunately, all the nuclear physics calculations to date do not yield a definitive answer.

\footnotetext{
${ }^{1}$ hfchau@hkusua.hku.hk.

2 Present address: Department of Physics, University of Hong Kong, Pokfulam Road, Hong Kong.
}

There are a number of difficulties in explaining some of the observational properties of pulsars using the strange star model. The first, and perhaps the most serious difficulty, is the possibility of a strange star glitch. Early EOS calculations suggested that strange stars have a very thin nuclear matter crust. The ratio of inertial moment of the nuclear matter crust to that of the whole strange star is of the order of $10^{-5}$. Moreover, the density of the nuclear matter crust is not high enough for neutron drip (Alcock et al. 1986; Alcock 1991). In contrast, pulsar glitch observations tell us that the ratio of inertial moment of pinned neutron superfluid crust to that of the whole star is about $10^{-2}$ (Alpar 1987; Link, Epstein, \& Van Riper 1992; Alpar et al. 1993). Nevertheless, by taking into account the existence of strange matter bound states, Benvenuto et al. (1991a, 1991b) showed that a strange star may support a "strange matter crust" thick enough to account for the observed pulsar glitches. Besides, a more recent calculation suggests that a strange star may be able to retain a reasonable size of nuclear matter crust by accretion as well (Benvenuto, Vucetich, \& Horvath 1994).

The second difficulty is the possibility of type I X-ray bursts on a strange star surface. It is commonly believed that type I X-ray bursts involve sudden thermonuclear runaway at the surface of an accreting neutron star (see, e.g., Lewin et al. 1991). The same bursting behavior cannot occur on a bare strange star surface because nuclear fuel will dissociate into constituent quarks immediately (Jones 1986). The possibility of X-ray bursts, therefore, requires the existence of a nuclear matter crust over a strange star. EOS calculations showed that the nuclear matter crust is separated from the interior strange matter by an electrostatic gap of thickness a few Fermi, thereby preventing the nuclear matter from converting into strange matter (Alcock et al. 1986; Benvenuto et al. 1991a). A strange pulsar magnetosphere can also be formed, giving rise to the observed radio pulsation (Alcock et al. 1986; Benvenuto et al. 1991b). 
The final difficulty is the magnetic field decay time. Assuming a normal strange matter core, Jones (1988) pointed that the magnetic field inside a strange star will not decay in a Hubble time. Moreover, there is no obvious mechanism for the star to retain a small residual field after say $10^{9} \mathrm{yr}$. Therefore, it is inconsistent with both the pulsar magnetic field decay hypothesis, and the spun-up formation scenario of millisecond pulsars (Jones 1988). Nevertheless, the statistical evidence for pulsar magnetic field decay over a period of some $10^{9} \mathrm{yr}$ is still inconclusive. Also, millisecond pulsars may rather form by accretion-induced collapse of white dwarfs (see, e.g., Bhattacharya \& Van den Heuvel 1991 and Bhattacharya \& Srinivasan 1995 for discussions on neutron star magnetic field decay). Therefore, the objection of Jones may not be completely well-founded. As I shall discuss in $\S 2$, the core of a strange star is likely to be superconducting. In this case, the magnetic field decay time estimated by Jones (1988) has to be modified. The question of magnetic field decay has been brought up in the review paper by Bailin \& Love (1984). They briefly mentioned that the low electron density in the strange stellar core may lead to rapid flux expulsion. However, they did not provide a detailed calculation. A major objective of this paper, therefore, is to perform such a calculation, taking into account the coupling between magnetic evolution and rotational dynamics of the star in the presence of collective effects such as clumping of flux tubes.

At this moment, one has no doubt that the standard neutron star model for pulsars is well tested by numerous observational data. However, we still have to take a close look at the alternative hypothesis, namely, the strange pulsar model (Benvenuto et al. 1991b). In particular, we would like to explore various physical properties of a neutron star and a strange star and to see if there are further ways to test which of the two models is a better candidate in explaining pulsar observations.

As I shall discuss in $\S 2$, the core of a strange star is likely to be superconducting (Bailin \& Love 1982, 1984). However, this creates a problem for the rotation of a strange star. In order to rotate an object with one superconducting species, a uniform magnetic field

$$
\boldsymbol{B}=\frac{2 m^{*} c}{q^{*}} \boldsymbol{\Omega},
$$

called the London field, is set up in the object (Baym 1988), where $m^{*}$ and $q^{*}$ are the effective mass and charge of the Cooper pair, and $\Omega$ is the rotational angular velocity of the object. But for a strange star, $u, d$, and $s$ quarks are all superconducting. These superconducting species require different values of $B$ to set up a rotation. Thus, we have a frustrated system. The situation is further complicated by the fact that all three superconducting quark flavors interact strongly with each other. In $\S 3$, I tackle this rotation problem using the Ginzburg-Landau formalism. Instead of setting up a uniform London field, I find that vortices and localized magnetic fields are created when the superconducting quarks rotate together. Then in $\S 4$, I point out that the vortices will interpin with the magnetic flux tubes, which alters the magnetic field evolution and rotation of a strange star dramatically. In particular, it suggests that the (superconducting) strange pulsar hypothesis is inconsistent with the observed magnetic field decay time in pulsars. Finally, a conclusion is drawn in $\S 5$.

\section{STRANGE MATTER SUPERCONDUCTIVITY}

Using a relativistic treatment of the BCS theory, Bailin \& Love $(1982,1984)$ suggested that strange matter turns superconducting at low temperatures. Using perturbative QCD at the one gluon exchange level, they showed that the pairing of quarks is most likely to occur in both the $u d-d u$ and the ss channels. The ss pairing is expected to have a gap matrix transforming as a color $\overline{3}$ and having $J^{P}=1^{+}$(and hence, the pairing is in $p$-wave). On the other hand, pairing in the $u-d$ system is expected to occur in the isoscalar channel, with a gap matrix transforming as a color 3 and having $J^{P}=0^{+}$(and hence, the pairing is in $s$-wave) (Bailin $\&$ Love 1984). The superconducting transition temperature is about $400 \mathrm{keV}$. Incidentally, the transition temperatures for neutron superfluid and proton superconductor in a neutron star core are of the same order of magnitude. Thus, about 1000 years after its supernova birth, the interior of a typical strange star is already cold enough for quark superconductivity (Benvenuto \& Vucetich 1991). However, one should notice that the above estimation depends quite sensitively on the quark-gluon coupling, and may also change if one goes beyond the one gluon exchange calculations. Thus, the existence of a superconducting core in a strange star is not completely conclusive even though it is very likely. Nonetheless, I assume the existence of quark superconductivity in a strange star core in this paper.

The quark superconductor is likely to be marginally type I with a zero temperature critical field $B_{c}$ about $10^{16} \mathrm{G}$ (Bailin \& Love 1984). Incidentally, the lower critical field of proton superconductor at the core of a neutron star is also of this order. The typical magnetic field of a canonical pulsar is about $10^{12} \mathrm{G}$, so naively one would expect complete flux expulsion from the superconducting strange matter core. However, the huge electrical conductivity of the normal state opposes the motion of the flux, thereby leading to a long flux expulsion time. In the case of a neutron star core, the flux expulsion time is $\sim 10^{8} \mathrm{yr}$ (Baym, Pethick, \& Pines 1969). This time is shorter for strange stars because of their low electron density and strong quarkquark scattering amplitude. Bailin \& Love (1982) predicted that it may be as little as $10^{4} \mathrm{yr}$. However, their estimate did not take into account the possibility of flux clumping, which may greatly increase the flux expulsion time. Moreover, if the flux expulsion rate is so fast, then a Meissner state is formed in an old pulsar. As we shall point out in $\S 4.4$, the thin crustal nuclear matter is not strong enough to support the magnetic stress and tension of the expelled field. Therefore, we believe that during the life of a canonical pulsar, a metastable state with magnetic field penetrating through the superconducting strange star core has to be formed. We shall return to this point later in $\S 4$.

Since the quark superfluid also couples to the color vector gluons, color superconductivity may also be observed. Because of color confinement outside the stellar core, we do not have the color analog of the ambient $10^{12} \mathrm{G}$ magnetic field (Bailin \& Love 1984). Thus, the observational consequences of color superconductivity remains unclear.

\section{ROTATING STRANGE STAR-GINZBURG-LANDAU APPROACH}

As I have discussed in $\S 1$, a London magnetic field is set up when a single species of superconducting sample is rotated (Baym 1988; Leggett 1991). In fact, the presence of 
London field has been observed in terrestrial superconductors (Cabrera 1987). In $\S 3.1$, we rederive the expression in equation (1) using Ginzburg-Landau formalism as a warm up to the multisuperconducting-component situation like that in a strange star. In the derivation, I find that there is a critical angular velocity above which the uniform London field state is not the most energetically stable configuration. This critical angular velocity, as far as I know, has not been investigated in the literature. Unfortunately, such a critical angular velocity is too high to be attained experimentally.

\subsection{The Case when There Is only One Superconducting Species}

We assume that the Cooper pairs in the sample are in the $s$ state. Thus, the order parameter for the only superconducting species $\Psi$ is complex. If the Cooper pairs are in a higher angular momentum state, the order parameter will be a complex matrix (Vollhardt \& Wölfle 1990). We, however, shall only stick to the simple $s$-wave pairing derivation. Similar results also apply to higher angular momentum pairing states although the derivation becomes rather complicated. For a sufficiently small angular velocity $\Omega$ and sample size, it is reasonable that the speed of the superfluid is not high at any point, and hence, a nonrelativistic treatment will suffice. In the corotating frame, the GinzburgLandau energy functional reads as $F=\int f d V$, where

$$
\begin{aligned}
f=- & a|\Psi|^{2}+\frac{b}{2}|\Psi|^{4}+\frac{1}{2 m^{*}} \\
& \times\left|\left(\frac{\hbar \boldsymbol{\nabla}}{i}+\frac{q^{*}}{c} \boldsymbol{A}-m^{*} \boldsymbol{\Omega} \times \boldsymbol{r}\right) \Psi\right|^{2}+\frac{1}{8 \pi}|\boldsymbol{\nabla} \times \boldsymbol{A}|^{2} .
\end{aligned}
$$

Here, $m^{*}$ and $q^{*}$ are the effective mass and charge of the Cooper pairs. Moreover, $a, b>0$ so that the superconducting state is preferred over the normal state. In general, $a$, and $b$ are temperature dependent. However, a few hundred years after its supernova birth the cooling timescale for a strange star is much longer than its spin-down timescale. Thus, as far as rotational dynamics of the star is concerned, we may assume that $a$ and $b$ are constants.

We consider the case when there is no external magnetic field. The presence of an external magnetic field may simply create extra fluxoids in the sample, which is not very interesting. There are two ways to minimize the kinetic energy term in equation (2). First, we can set up a magnetic field so that the $\boldsymbol{\Omega} \times \boldsymbol{r}$ contribution is canceled by $\boldsymbol{A}$. Alternatively, we can create normal cores (i.e., line defects in the form of vortices similar to that of a rotating superfluid). However, there are prices to pay for both cases: setting up a magnetic field requires magnetic energy; setting up a vortex needs both a kinetic energy near the vortex and a nucleation energy for creation of the vortex core. What really happens in the sample, in general, is that a uniform magnetic field together with an array of vortices will be formed when it is rotated. Suppose a uniform magnetic field $\boldsymbol{B}$ (parallel to $\boldsymbol{\Omega}$ ) is set up in the star, then $\boldsymbol{A}=\boldsymbol{B} \times \boldsymbol{r} / 2$. Then in order to minimize the kinetic energy term in equation (2), vortices (or antivortices) have to be formed just like a rotating superfluid (see, e.g., Sauls 1989). Unlike fluxoids, vortices carries circulation but not magnetic field. The required number of vortices (or antivortices) per unit area is given by

$$
n(B)=\frac{2}{\kappa}\left|\Omega-\frac{q^{*}}{2 m^{*} c} B\right|,
$$

where $\kappa=h / m^{*}$ is the circulation quantum. Obviously, vortices form an Abrikosov lattice in the absence of spatial inhomogeneity. The Ginzburg-Landau free energy per unit volume of the system is, therefore, given by (Sauls 1989; Vollhardt \& Wölfle 1990)

$$
\frac{F}{V} \approx \frac{B^{2}}{8 \pi}+\left\{\frac{\kappa^{2} \rho_{s}}{4 \pi}\left[\ln \left(\frac{R_{c}}{\xi}\right)-\frac{3}{4}\right]+\frac{1}{2} \pi \xi^{2} N(0) \Delta^{2}\right\} n(B),
$$

where $\rho_{s}=m \Psi * \Psi$ is the density of superconducting species, $\xi$ is the coherence length of the vortex, $R_{c}$ is the upper cutoff length, which is of the order of the intervortex spacing, $\Delta$ is the superconducting gap energy, and $N(0)$ is the density of state for one spin projection on the Fermi surface, which is given by

$$
N(0)=\left[k^{2} \frac{d k}{d E_{k}}\right]_{k=k_{F}} \approx \frac{E_{F}^{3}}{2 \pi^{2} \hbar^{3} c^{3}}
$$

in the extreme relativistic limit.

Physically, the first term in equation (4) is the magnetic energy, and the second term is the kinetic and nucleation energies of the vortices. By minimizing equation (4), we find that the magnetic field generated in the star is

$$
B=\left\{\begin{array}{lc}
\frac{2 m^{*} c}{q^{*}} \Omega & \text { if } \Omega<\Omega_{c}, \\
\frac{q^{*}}{m^{*} c \kappa}\left\{\kappa^{2} \rho_{s}\left[\ln \left(\frac{R_{c}}{\xi}\right)-\frac{3}{4}\right]+2 \pi^{2} \xi^{2} N(0) \Delta^{2}\right\} & \text { otherwise },
\end{array}\right.
$$

where we have neglected the dependence of $\ln R_{c}$ on $B$ because it is rather weak. The critical angular velocity $\Omega_{c}$ above which vortices begin to form and the translational symmetry of the system is spontaneously broken can be determined from equations (1) and (6). And it is given by

$$
\Omega_{c}=\frac{q^{* 2} \rho_{s} h}{2 m^{* 3} c^{2}} .
$$

Since magnetic energy scales quadratically with magnetic field while the energy required to form an array of vortices scales linearly with it, formation of vortices is favored at high rotational rates. For a typical terrestrial superconductor (high or low $T_{c}$ ), the effective charge and mass of the Cooper pairs are given by $q^{*}=-2 e$ and $m^{*}=2 m_{e}$, respectively. By putting $\rho_{s} \sim 10^{-6} \mathrm{~g} \mathrm{~cm}^{-3}$, we obtain $\Omega_{c} \sim$ $6 \times 10^{8} \mathrm{rad} \mathrm{s}^{-1}$. Similarly, for neutron star matter, $q^{*}=2 e$, $m^{*}=2 m_{p}$, and $\rho_{s} \sim 2 \times 10^{12} \mathrm{~g} \mathrm{~cm}^{-3}, \Omega_{c} \sim 2 \times 10^{17} \mathrm{rad}$ $\mathrm{s}^{-1} . \Omega_{c}$ for strange star matter is similar to that of a neutron star. These angular velocities, unfortunately, are so high that the sample or the star will break apart well before the formation of vortices. In other words, we can neglect the magnetic energy contribution in the Ginzburg-Landau free energy all the time.

\subsection{The Case when There Are Multiple Noninteracting Superconducting Species}

Now we examine the case when there is more than one superconducting species. We consider the simple case when they are noninteracting. Thus, the only coupling between them is their response to the same vector potential $\boldsymbol{A}$. If we label each superconducting species by $j$, the Ginzburg- 
Landau free energy density is

$$
\begin{aligned}
f & =\sum_{j}\left\{-a_{j}\left|\Psi_{j}\right|^{2}+\frac{b_{j}}{2}\left|\Psi_{j}\right|^{4}+\frac{1}{2 m_{j}^{*}}\right. \\
& \left.\times\left|\left(\frac{\hbar \nabla}{i}+\frac{q_{j}^{*}}{c} \boldsymbol{A}-m_{j}^{*} \boldsymbol{\Omega} \times \boldsymbol{r}\right) \Psi_{j}\right|^{2}\right\}+\frac{1}{8 \pi}|\nabla \times \boldsymbol{A}|^{2} .
\end{aligned}
$$

Once again, we expect each superconducting species to form vortices in addition to the generation of a common magnetic field. (Compare with Mendell \& Lindblom 1991 for a similar study using hydrodynamics.) As we have shown in $\S 3.1$, we can neglect the magnetic energy contribution. As a result, for a uniform magnetic field $B$, the Ginzburg-Landau free energy per unit volume becomes

$$
\begin{aligned}
\frac{F}{V} \approx \sum_{j}\left\{\frac{\hbar \rho_{s j}}{m_{j}^{*}}\left[\ln \left(\frac{R_{c j}}{\xi_{j}}\right)-\frac{3}{4}\right]\right. \\
\left.+\frac{m_{j}^{*} \xi_{j}^{2}}{2 \hbar} N_{j}(0) \Delta_{j}^{2}\right\}\left|\Omega-\frac{q_{j}^{*}}{2 m_{j}^{*} c} B\right| .
\end{aligned}
$$

As shown in Figure 1, for a fixed $\Omega$, the free energy density contribution of each superconducting species assumes its minimum value when $B$ equals its London magnetic field. Neglecting the weak dependence of $\ln R_{c}$ on $B, F / V$ is a continuous and piecewise linear function of $B$ with "vertices" locate at the points where $B$ equals the London magnetic field of any one of its superconducting species. Thus, the actual magnetic field $B$ set up in the sample always equals a London magnetic field of a particular superconducting species. In other words, at least one superconducting species rotates without creating vortices. While other species with a different London field have to create vortices (or antivortices) in order to rotate with the same angular velocity. This finding is somewhat unexpected because one would naively think that the value of the magnetic field set up in the sample would be a compromise between different London fields and all superconducting species would form vortices. As the superconducting species do not interact, vortices from each species will form Abrikosov lattices on their own (in the absence of spatial inhomogeneity).

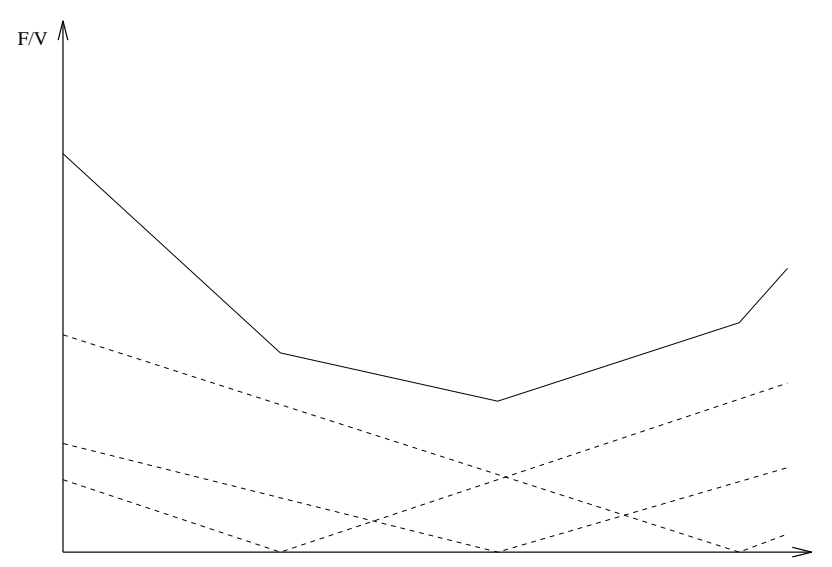

B

Fig. 1.-Schematic plot of $F / V$ as a function of $B$ for a fixed $\Omega$ in a multisuperconducting noninteracting species sample. Dashed lines represent the contributions from individual superconducting species, and the solid line is their combined free energy per unit volume.
What can we predict if the quarks in strange stars were noninteracting? Since the masses (bare or constituent) of $d$ and $u$ are about the same, which are both much less than that of $s$, equation (1) tells us that their corresponding London fields satisfy

$$
B_{s}<0<B_{u d},
$$

when $\Omega>0$. (An exactly reverse relationship is true when $\Omega<0$.) Since $\rho_{u} / m_{u}^{*} \approx \rho_{d} / m_{d}^{*} \gg \rho_{s} / m_{s}^{*} \quad$ (Alcock 1991), equation (8) tells us that $g_{u d} \gg g_{s}$, where

$$
g_{j}=\frac{\rho_{j}\left|q_{j}^{*}\right|}{2 m_{j}^{* 2} c} \text { for } j=u d, s .
$$

Combining equations (9), (10), and (11), the slope $M$ of our piecewise linear free energy density curve $F / V$ is given by

$$
M= \begin{cases}g_{s}+g_{u d}>0 & \text { for } B>B_{u d}, \\ g_{s}-g_{u d}<0 & \text { for } B_{s}<B<B_{u d}, \\ -g_{s}-g_{u d}<0 & \text { for } B<B_{s},\end{cases}
$$

where we have neglected the small nucleation energy contribution in the above estimate.

Consequently, $F / V$ finds its minimum when $B=B_{u d}$. (Readers can verify that this conclusion is independent of the sign of $\Omega$.) So if different flavor quarks were noninteracting, only $s$ quarks would form vortices upon rotation.

\subsection{The Case when There Are Multiple Interacting Superconducting Species}

Finally, we consider the (realistic) case when the quarks are interacting. The form of the free energy density must be invariant under global phase changes in any of the order parameters. If we consider terms up to second order in gradients and quartic in order parameters, the most general form of $f$ is given by (compare with Alpar, Langer, \& Sauls 1984b, where they have omitted the quartic self-interaction terms)

$$
\begin{aligned}
& f=\frac{1}{8 \pi}|\nabla \times \boldsymbol{A}|^{2}+\sum_{j}\left\{-a_{j}\left|\Psi_{j}\right|^{2}+\frac{1}{2 m_{j}^{*}}\left|\mathscr{P}_{j} \Psi_{j}\right|^{2}\right\} \\
& +\frac{1}{2} \sum_{j, k}\left\{b_{j k}\left|\Psi_{j}\right|^{2}\left|\Psi_{k}\right|^{2}+\mu_{j k}\left(\mathscr{P}_{j} \Psi_{j}^{*}\right) \cdot\left(\mathscr{P}_{k} \Psi_{k}\right) \Psi_{k}^{*} \Psi_{j}\right. \\
& \left.+v_{j k}\left(\mathscr{P}_{j} \Psi_{j}^{*}\right) \cdot\left(\mathscr{P}_{k} \Psi_{k}^{*}\right) \Psi_{j} \Psi_{k}+v_{j k}^{*}\left(\mathscr{P}_{j} \Psi_{j}\right) \cdot\left(\mathscr{P}_{k} \Psi_{k}\right) \Psi_{j}^{*} \Psi_{k}^{*}\right\},
\end{aligned}
$$

where $b$ is a real symmetric matrix, $\mu$ is a hermitian matrix, and

$$
\mathscr{P}_{j}=\frac{\hbar \boldsymbol{\nabla}}{i}+\frac{q_{j}^{*}}{c} \boldsymbol{A}-m_{j}^{*} \boldsymbol{\Omega} \times \boldsymbol{r}
$$

is the covariant momentum operator in the corotating frame for the $j$ th species. Since the free energy has to be bounded from below, we further require all the eigenvalues of the symmetric matrix $b$ to be positive.

To explicitly show the drag effect of one superconducting species on the other, we write the order parameters $\Psi_{j}$ as $\left|\Psi_{j}\right| \exp \left(i \varphi_{j}\right)$, where $\varphi_{j}$ are the phases of the order parameters. Then the velocity of the superconducting species $j$ in the rotating frame is given by

$$
\boldsymbol{v}_{j}=\frac{1}{m_{j}^{*} \Psi_{j}} \mathscr{P}_{j} \Psi_{j} \approx \frac{\hbar}{m_{j}^{*}} \nabla \varphi_{j}+\frac{q_{j}^{*}}{m_{j}^{*} c} \boldsymbol{A}-\boldsymbol{\Omega} \times \boldsymbol{r},
$$


where we have neglected the spatial variation of $\left|\Psi_{j}\right|$. From equations (13) and (15), we have

$$
\begin{aligned}
f= & \frac{1}{8 \pi}|\nabla \times \boldsymbol{A}|^{2}-\sum_{j} a_{j}\left|\Psi_{j}\right|^{2} \\
& +\frac{1}{2} \sum_{j, k}\left[b_{j k}\left|\Psi_{j}\right|^{2}\left|\Psi_{k}\right|^{2}+\rho_{j k} v_{j} \cdot v_{k}\right],
\end{aligned}
$$

where

$$
\rho_{j j}=m_{j}^{*}\left|\Psi_{j}\right|^{2}+\mu_{j j} m_{j}^{* 2}\left|\Psi_{j}\right|^{4}+2 v_{j j} m_{j}^{* 2}\left|\Psi_{j}\right|^{4}
$$

and

$$
\rho_{j k}=\left(\mu_{j k}+2 v_{j k}\right) m_{j}^{*} m_{k}^{*}\left|\Psi_{j}\right|^{2}\left|\Psi_{k}\right|^{2}
$$

for $j \neq k$. Thus, once a superconducting species moves, equation (16) tells us that in general it is energetically favorable for the other superconducting species to move along with it as well. A similar conclusion can be reached by using three-velocity hydrodynamics (Khalatnikov 1957; Andreev \& Bashkin 1976; Vardanyan \& Sedrakyan 1981).

At the strange matter density, which is about $5 \times 10^{14} \mathrm{~g}$ $\mathrm{cm}^{-3}$, the "fine-structure constant" for strong force $\alpha_{s}$ is about 0.5-0.6 (Benvenuto et al. 1991b) indicating that QCD is the dominant interaction between the quark Cooper pairs. The values of the off-diagonal terms of the matrix $\rho$, which measure the strength of the drag, can be calculated (in principle) from the QCD interaction Hamiltonian. Although the large value of $\alpha_{s}$ prevents us from using perturbation theory, we believe that the effective coupling constants $\mu_{i j}$ and $v_{i j}$ in equation (13) are at least of order of $\alpha_{s}$. Thus, the drag force between different quark flavors are so strong that up to first-order approximation, all three quark flavors move at the same velocity except possibly near the superfluid cores. The comoving approximation greatly simplifies our effort to find the minimum system configuration of a strange star.

In order to minimize the drag energy, all the superconducting species have to share common normal cores. We call such a configuration a vortex bundle. The circulation of the $j$ th superconducting species around a circle of radius $r$ centered at the axis of rotation of the star in the corotating frame is given by

$$
\begin{aligned}
0 & =\oint v_{j} \cdot d \boldsymbol{l} \\
& =\oint \frac{\hbar}{m_{j}^{*}} \nabla \varphi_{i} d \boldsymbol{l}+\frac{q_{j}^{*}}{m_{j}^{*} c} \int \boldsymbol{B} \cdot d \boldsymbol{S}-\int \nabla \times(\boldsymbol{\Omega} \times \boldsymbol{r}) \cdot d \boldsymbol{S} \\
& =\frac{h N_{j} L}{m_{j}^{*}}+\frac{q_{j}^{*} \Phi_{v} L}{m_{j}^{*} c}+\frac{q_{j}^{*} \pi r^{2} B_{g}}{m_{j}^{*} c}-2 \pi r^{2} \Omega,
\end{aligned}
$$

where $\Omega$ is the angular speed of the strange star seen by an external inertial observer, $N_{j}$ is the number of vortex quantum per bundle for species $j, L$ is the number of vortex bundles, $\Phi_{v}$ is the magnetic flux in the core of a vortex bundle, and $B_{g}$ is the global uniform (London) magnetic field in the star. Thus, the vortex bundle density $\mathscr{D}$ is given by

$$
\mathscr{D}\left(h N_{j}+\frac{q_{j}^{*} \Phi_{v}}{c}\right)=2 m_{j}^{*} \Omega-\frac{q_{j}^{*} B_{g}}{c} \text { for all } j .
$$

Using the same idea, we can show that the speed of superconducting species $j$ at a small distance $r$ from the core of a vortex bundle as seen in the corotating frame is given by

$$
v_{j}(r)=\frac{1}{2 \pi m_{j}^{*} r}\left(h N_{j}+\frac{q_{j}^{*} \Phi_{v}}{c}\right)+\frac{q_{j}^{*} B_{g} r}{2 m_{j}^{*} c}-\Omega r \text { for all } j .
$$

Thus, the comoving requirement of supercurrents at all spatial points requires that (1) $q_{j}^{*} B_{g} / 2 m_{j}^{*} c-\Omega$ is a constant for all $j$, which is possible only if the global uniform London field $B_{g}$ is zero; and (2) $h N_{j} / m_{j}^{*}+q_{j}^{*} \Phi_{v} / m_{j}^{*} c$ is a constant for all $j$, implying that the integers $N_{j}$, and the magnetic flux $\Phi_{v}$ are chosen in such a way that

$$
\frac{h N_{j}}{m_{j}^{*}}+\frac{q_{j}^{*} \Phi_{v}}{m_{j}^{*} c}=K
$$

for some constant $K \neq 0$ for all $j$. $K$ can be interpreted as the circulation of a vortex bundle in this strongly interacting superconducting system.

The necessary and sufficient conditions for the existence of solutions to equation (21) are proven in Appendix A. It turns out that the comoving constraint (eq. [21]) is very stringent: In general, solution may not exist for a system involving more than two species of superconducting Cooper pairs. And in the case where a solution of equation (21) does not exist, the only way out is that superconductivity in some species are destroyed. Luckily, in the case of strange star matter, there are only two species of Cooper pairs, namely, $u d-d u$ and ss, so that the solution in the strongly interacting limit exists. In this limit, we expect all the quark flavors to form quantized vortices when the star rotates. The normal vortex core of each superconducting species shares a common region of space, in the form of vortex bundles. A magnetic field may be present in the vortex bundle cores. Moreover, stellar rotation is made possible by the formation of vortex bundles rather than by a uniform London magnetic field.

In case of the strange star matter, the $u d-d u$ and ss Cooper pairs have charges $q_{u d}^{*}=e / 3$ and $q_{s}^{*}=-2 e / 3$, respectively. Also, the effective mass of a strange quark at the strange star interior $m_{s} \sim 175 \mathrm{MeV}$ (Benvenuto et al. 1991b). Therefore, the effective mass of an ss Cooper pair, $m_{s}^{*}$, is approximately $350 \mathrm{MeV}$. The effective mass of a $u$ or $d$ quark, $m_{u d}$, is of order of $10 \mathrm{MeV}$, giving $m_{u d}^{*} \sim 20 \mathrm{MeV}$. Since $\left(m_{u d}, q_{u d}\right)$ and $\left(m_{s}, q_{s}\right)$ are linearly independent, the solution of equation (21) is given by (see Appendix A)

$$
\left(\begin{array}{c}
K \\
\Phi_{v}
\end{array}\right)=\frac{h}{\left(m_{s}^{*} q_{u d}^{*}-m_{u d}^{*} q_{s}^{*}\right)}\left[\begin{array}{c}
q_{u d}^{*} N_{s}-q_{s}^{*} N_{u d} \\
c\left(m_{u d}^{*} N_{s}-m_{s}^{*} N_{u d}\right)
\end{array}\right],
$$

where $N_{u d}$ and $N_{s}$ are the number of quanta per vortex bundle for $u d-d u$ and ss Cooper pairs, respectively, and $K \neq 0$. In the zero temperature limit, the system will choose the ground state configuration out of the above infinitely many solutions. Similar to equation (4), the GinzburgLandau free energy per unit volume in the strongly interacting limit is given by

$$
\begin{aligned}
\frac{F}{V} \approx & \llbracket \sum_{j}\left\{\frac{h^{2} \rho_{s j} N_{j}^{2}}{4 \pi m_{j}^{* 2}}\left[\ln \left(\frac{R_{c j}}{\xi_{j}}\right)-\frac{3}{4}\right]\right. \\
& \left.+\frac{\pi \xi_{j}^{2}}{2} N_{j}(0) \Delta_{j}^{2}\right\}+\frac{\Phi_{v}^{2}}{8 \pi^{2} \lambda^{2}} \rrbracket|\mathscr{D}|,
\end{aligned}
$$


where $\lambda$ is the penetration depth, and $\mathscr{D}$ is the number density of vortex bundles. From equation (19), $\mathscr{D}\left(h N_{j}\right.$ $\left.+q_{j}^{*} \Phi_{v} / c\right)=2 m_{j}^{*} \Omega$ in the strongly interacting limit. Consequently,

$$
\begin{aligned}
\frac{F}{V} \approx & \llbracket \sum_{j} \frac{1}{h N_{j} c+q_{j}^{*} \Phi_{v}}\left\{\frac{h^{2} \rho_{s j} N_{j}^{2}}{2 \pi m_{j}^{*}}\left[\ln \left(\frac{R_{c j}}{\xi_{j}}\right)-\frac{3}{4}\right]\right. \\
& \left.\left.+\pi m_{j}^{*} \xi_{j}^{2} N_{j}(0) \Delta_{j}^{2}\right\}+\frac{\Phi_{v}^{2} m_{1}^{*}}{4 \pi^{2} \lambda^{2}\left(h N_{1} c+q_{1}^{*} \Phi_{v}\right)}\right]|c| \Omega \mid
\end{aligned}
$$

increases (approximately) linearly with the angular speed $|\Omega|$ of the star.

Now, we estimate the average free energy density for strange star matter. BCS theory tells us that $\Delta=1.76 k T_{c}$ for s-wave paired superconductor. Putting $k T_{c} \sim 400 \mathrm{keV}$ for strange stellar matter (Bailin \& Love 1984), we find $\Delta_{u d} \sim$ $700 \mathrm{keV}$. The number densities for $u$ and $d$ quarks are given by (Alcock et al. 1986)

$$
n_{j}=\frac{1}{\pi^{2}}\left(1-\frac{2 \alpha_{s}}{\pi}\right) \mu_{j}^{3} \text { for } j=u, d,
$$

where $\mu_{j}$ is the chemical potential. Putting $\alpha_{s}=0.5$ and $\mu_{u} \approx \mu_{d} \sim 400 \mathrm{MeV}$ (Benvenuto et al. 1991b), we obtain $n_{u} \approx n_{d} \sim 5.7 \times 10^{38} \mathrm{~cm}^{-3}$. Since the electron number density is much less than that of the quarks (Bailin \& Love 1984; Alcock et al. 1986; Benvenuto et al. 1991b), the charge neutrality condition reads

$$
\frac{2}{3} n_{u}-\frac{1}{3} n_{d}-\frac{1}{3} n_{s}=n_{e} \approx 0 .
$$

Therefore, we expect $n_{s} \approx n_{d}$.

For both $u$ and $d$ quarks, $E_{F} \approx \mu \sim 400 \mathrm{MeV}$ (Bailin \& Love 1984), and hence from equation (5), $N_{u d}(0) \sim 6.6$ $\times 10^{41} \mathrm{erg}^{-1} \mathrm{~cm}^{-3}$. Also, $m_{u} \approx m_{d} \sim 10 \mathrm{MeV}$. The coherence length $\xi_{j}$ can be estimated in the framework of relativistic Ginzburg-Landau theory. Bailin \& Love (1984) argue that

$$
\xi_{j}^{3} \approx \frac{7 \zeta(3)}{2 k T_{c_{j}} p_{F_{j}} \mu_{j}},
$$

where $\zeta(3) \sim 1.20$ and $p_{F_{j}}$ is the Fermi momentum of species $j$, which is approximately equal to $E_{F_{j}} / c$ inside a strange star. Thus, $\xi_{u d} \sim 8.0 \mathrm{fm}$. Since the quark superconductor is likely to be marginally type $\mathrm{I}, \lambda \approx 2^{1 / 2} \xi_{u d} \sim 11.3 \mathrm{fm}$. Assuming $\ln \left(R_{c j} / \xi_{j}\right)$ to be of the order of 10 , I numerically compute the average free energy density in equation (24) for all possible values of $N_{u d}$ and $N_{s}$. I find that the ground state configuration for strange star matter is achieved when $N_{s}=1, N_{u d}=0$. Consequently, the values of $K$ and $\Phi_{v}$ in the ground state configuration are given by

$$
K=\frac{h}{m_{s}^{*}-2 m_{u d}^{*}} \sim 0.012 \mathrm{~cm}^{2} \mathrm{~s}^{-1}
$$

and

$$
\Phi_{v}=\frac{3 h c m_{u d}^{*}}{e\left(m_{s}^{*}-2 m_{u d}^{*}\right)} \sim 8.0 \times 10^{-8} \mathrm{G} \mathrm{cm}^{2},
$$

respectively.

In summary, the star behaves quite differently in the noninteracting and strongly interacting limits. In the noninteracting limit, a global magnetic field is set up, and vortices are formed in all but one superconducting species when the system rotates. In contrast, vortex bundles in the form of Abrikosov lattice is present in a rotating strongly interacting system. Moreover, the global uniform London magnetic field is no longer present. Regarding the coupling between the quarks as a tuning parameter, it is interesting to map out the phase diagram of this system. This will be carried out in future works.

\section{VORTEX INTERPINNING AND ITS ASTROPHYSICAL CONSEQUENCES}

The formation of vortices in a rotating superconducting strange star has at least three possible observational consequences if one assumes that the observed pulsars are in fact strange stars instead of neutron stars. First, the heat capacity of the star and the cooling processes are modified and its effect on the strange star cooling has been studied (Benvenuto \& Vucetich 1991; Page 1992). In this section, we concentrate on the second effect, namely, the magnetic field decay due to interpinning of vortex bundles and the magnetic fluxoids. I shall discuss briefly possible magnetic field alignment due to interpinning in $\S 4.2$ as well.

Nucleation energy is required in the creation of normal cores in both vortex bundles and magnetic fluxoids in a strange star. Therefore, it is energetically favorable for a vortex bundle to "pin" to a magnetic fluxoid so that a lesser volume of normal strange matter has to be nucleated. Thus, the dynamics of vortex bundles and magnetic flux tubes are coupled together. Similar to the case of superfluid neutron vortices in a rotating neutron star (Alpar et al. 1993), equation (24) tells us that in the strongly interacting limit, the quark vortex bundle density is directly proportional to the angular speed $|\Omega|$ of the rotating strange star. As a strange star spins down, its vortex bundles in the star core have to move radially outward from the rotational axis (and eventually some of them will annihilate near the stellar surface). The pinning of quark vortex bundles and magnetic flux tubes implies that the rotational and magnetic evolution of a strange star are coupled.

Alternatively, the vortex bundle and the flux tubes can pin together by interaction of their core magnetic fields. When two magnetic carrying wires are placed together, an energy change of $\boldsymbol{B}_{1} \cdot \boldsymbol{B}_{2} V / 4 \pi$ is expected, where $V$ is their interacting volume, and $\boldsymbol{B}_{j}$ are their magnetic field strength. Depending on their relative field orientation, the two wires experience either mutual attraction or repulsion. In the former case, the wires will pin to each other; and in the latter case, the wires will avoid each other, and hence, effectively "pin" to the interwire spaces. In fact, similar ideas have been applied to the proton fluxoids and magnetic vortices in neutron star cores; and their possible observational consequences have been studied (Sauls 1989; Srinivasan et al. 1990; Chau, Cheng, \& Ding 1992; Ding, Cheng, \& Chau 1993).

\subsection{Estimation of Pinning Energy per Intersection}

Now we estimate the pinning energy per intersection of a vortex bundle with a flux tube by both pinning mechanisms. The pinning energy due to nucleation per intersection is given by

$$
\left(E_{p}\right)_{\mathrm{nucl}} \approx \frac{1}{2} \sum_{j} N_{j}(0) \Delta_{j}^{2} V_{j}
$$


The intersection volume for species $j, V_{j}$, is given by

$$
V_{j} \approx \alpha \pi \mathscr{N}_{\mathrm{flux}}^{1 / 2} \xi_{j}^{3},
$$

where $\xi_{j}$ is the coherence length for species $j, \mathscr{N}_{\text {flux }}$ is the number of flux quantum in a flux tube, and $\alpha$ is a geometrical factor depending on the angle between the magnetic flux tube and the vortex, $\theta$, and their elastic moduli. For stiff magnetic flux tubes and vortex bundles, $\alpha \approx 2 \operatorname{cosec}|\theta|$.

We estimate the nucleation pinning energy as follows: for an s-wave superconductor, BCS theory tells us that $\Delta=$ $1.76 k T_{c}$; and for a $p$-wave superconductor, $\Delta=2.4 k T_{c}$ (Baym \& Pethick 1975). Putting $k T_{c} \sim 400 \mathrm{keV}$ for strange stellar matter (Bailin \& Love 1984), we obtain $\Delta_{u d} \sim 700$ $\mathrm{keV}$ and $\Delta_{s} \sim 960 \mathrm{keV}$. For $u d-d u$ quark Cooper pairs, $E_{F} \approx \mu \sim 400 \mathrm{MeV}$ (Bailin \& Love 1984). And beta equilibrium requires $E_{F_{s}} \approx \mu_{s}=\mu_{d} \approx 400 \mathrm{MeV}$. The coherence lengths are given by equation (27), giving us $\xi_{u d} \approx \xi_{s} \sim 8 \mathrm{fm}$. So from equations (5), (29), and (30), we obtain

$$
\left(E_{p}\right)_{\text {nucl }} \sim 2.4 \mathscr{N}_{\text {flux }}^{1 / 2} \operatorname{cosec}|\theta| \mathrm{MeV} .
$$

Similarly, the magnetic pinning energy is given by (Chau et al. 1992; Ding et al. 1993)

$$
\left(E_{p}\right)_{\mathrm{mag}} \approx \frac{\left|\boldsymbol{B}_{\text {vortex }} \cdot \boldsymbol{B}_{\text {fluxoid }}\right|}{4 \pi} V \approx \frac{B_{c} \Phi_{v} \lambda \mathcal{N}_{\text {flux }}^{1 / 2}}{2 \pi}|\cot \theta|,
$$

where $B_{c}$ is the critical magnetic field. Since the flux quantum for a strange star flux tube equals $h c / 2(2 e /$ $3)=3 h c / 4 e$, and $\lambda \sim 11.3 \mathrm{fm}$, we obtain $B_{c} \sim 7.7 \times 10^{16} \mathrm{G}$. By putting the value of $\Phi_{v}$ obtained in equation (28b), we find

$$
\left(E_{p}\right)_{\text {mag }} \sim 690 \mathscr{N}_{\text {flux }}^{1 / 2}|\cot \theta| \mathrm{MeV} .
$$

So, unless the magnetic flux tubes and the vortices are perpendicular to each other (this happens when the rotational and magnetic axes of the star are orthogonal to each other), the magnetic pinning energy is approximately 2 orders of magnitude larger than the nucleation pinning energy. Therefore, we shall use the magnetic pinning energy in our subsequent calculations.

\subsection{Forces Acting on Flux Tubes and Vortices}

Following Ding et al. (1993), the major forces acting on flux tubes and vortices of a spinning down strange star are summarized below.

Interpinning force.- Since it is energetically favorable for the magnetic flux tubes and the vortex bundles to pin with each other, a force of order of $E_{p} / \xi$ is experienced by both parties when one tries to pull them apart. (Similar assertion is true when the magnetic pinning force is repulsive.) This interpinning force encourages the magnetic flux tubes and vortex bundles to move together.

Thermal activation.- Since $E_{p}$ is of order of $100 \mathrm{MeV}$ and the interior temperature of the star is $\lesssim 10 \mathrm{keV}$, random thermal noise can be an important source of "force." In particular, thermal activation can depin a vortex with a flux line.

Magnus force.-The magnetic flux tubes couple to the thin crust of the strange star via electromagnetic interaction between its strong core magnetic field and the background electron plasma. Therefore, the flux tubes corotate, and hence spin down, with the crust of the star. The vortex bundle core, due to interpinning, may spin down with the crust as well. equation (19) tells us that the only way to slow down the angular speed of a rotating superconducting quark fluid is by reducing its vortex bundle density. This can in turn be achieved only by moving the vortex bundles radially outward from the axis of rotation of the star. So as the star slows down, a steady angular velocity difference between the superconducting quark fluid and the crust is developed. Consequently, a hydrodynamic force, called the Magnus force, acting on the vortex bundles is developed. The Magnus force tries to push the vortex bundle radially outward from the rotational axis of the star, thereby reducing the angular velocity difference between the superconducting quark fluid and the crust. The combined effect of the Magnus force and the interpinning force may cause a spin-down-induced magnetic field expulsion in a strange star. The Magnus force also defines a preferred direction for the thermal activation (i.e., thermally assisted creeping). For an interwound network of pinned magnetic flux tubes and quark vortex bundles, the average force per unit length acting on a magnetic flux tube due to the Magnus force acting on the vortex bundles is given by (compare with Ding et al. 1993)

$$
f_{f, \mathrm{Mag}} \approx \frac{L_{v}}{L_{f}} \rho K r \omega \hat{\boldsymbol{e}}_{r},
$$

where $r$ is the distance from the rotational axis, $\omega$ is the angular velocity difference between the superconductor $\left(\Omega_{s}\right)$ and the stellar crust $\left(\Omega_{c}\right), K$ is the circulation given by equation (28a), $\rho$ is the total matter density, and $L_{f}$ and $L_{v}$ are the total number of flux tubes and vortex bundles, respectively, in the star. In fact, $L_{f}$ and $L_{v}$ can be determined by considering the total magnetic flux and the circulation of superconducting current of the star. They are given by

$$
L_{f}=\frac{4 \pi e R_{\text {star }}^{2}\left|B_{\text {star }}\right|}{3 h c \cdot \mathscr{N}_{\text {flux }}}
$$

and

$$
L_{v}=\frac{2 \pi R_{\mathrm{star}}^{2}|\Omega|}{K} \equiv \frac{4 \pi R_{\mathrm{star}}^{2}\left(m_{s}-2 m_{u d}\right)}{h}|\Omega|,
$$

where $R_{\text {star }}$ and $B_{\text {star }}$ are the radius and total magnetic field strength of the star, respectively.

The angular velocity difference, $\omega$, is likely to remain at its steady state value, which can be deduced from the vortex creep theory (Alpar et al. 1984a; Alpar et al. 1993) and is given by

$$
\begin{aligned}
\omega_{\infty} & = \pm \frac{k T}{\rho K b_{p} r \lambda} \sinh ^{-1}\left[\frac{r}{4 \Omega v_{0}}|\dot{\Omega}| \exp \left(\frac{E_{p}}{k T}\right)\right] \\
& \approx \pm \frac{E_{p}}{\lambda \rho K b_{p} r} \\
& \approx \pm \frac{B_{c} \Phi_{v}|\cos \theta|}{\pi \rho K r}\left(\frac{e\left|B_{\text {star }}\right|}{3 h c}\right)^{1 / 2}= \pm \omega_{\text {cr }},
\end{aligned}
$$

where we have assumed that $E_{p} \gg k T$. Here $v_{0} \sim 10^{13} \mathrm{~cm}$ $\mathrm{s}^{-1}$ is the microscopic creeping speed, $b_{p} \approx(1 /|\sin \theta|)$ $\times\left(\pi R_{\mathrm{star}}^{2} / L_{f}\right)^{1 / 2}$ is the mean distance between successive pinning sites along a vortex bundle, and $\omega_{\mathrm{cr}}$ is the critical angular velocity lag above which it is no longer energetically favorable for the vortices and fluxoids to pin together. The plus (or minus) sign is taken if the outward moving 
speed of vortex bundles is greater (or less) than that of magnetic flux tubes. Therefore, the maximum possible average force per unit length vortex bundles can exert on a flux tube equals $\rho K R_{\text {star }} \omega_{\mathrm{cr}} L_{v} / L_{f}$.

Finally, if the spin down of the star is mainly due to its dipole radiation lost, then the outward moving speed of the vortex bundles is given by (Ding et al. 1993)

$$
v_{v}(t) \approx \frac{B_{\mathrm{star}}^{2} R_{\mathrm{star}}^{7} \Omega^{2} \sin ^{2} \theta}{3 I c^{3}},
$$

where $I$ is the moment of inertia of the star.

Buoyancy force.-The presence of magnetic stress in the core of a flux line decreases the internal density of quark matter (Muslimov \& Tsygan 1985; Harvey, Ruderman, \& Shaham 1986; Jones 1987). Thus, the buoyancy force per unit length experienced by a flux line is

$$
f_{\text {buoy }}=\frac{9 h^{2} c^{2} \mathscr{N}_{\text {flux }} g}{128 e^{2} \pi^{2} \lambda^{2} c_{s}^{2}} \hat{\boldsymbol{e}}_{r} \approx \frac{9 h^{2} c^{2} \mathscr{N}_{\text {flux }}}{128 e^{2} \pi^{2} \lambda^{2} R_{\text {star }}} \hat{\boldsymbol{e}}_{r}
$$

where $g$ is the local acceleration due to gravity and $c_{s}^{2} \equiv$ $d P / d \rho \approx g R_{\text {star }}$ is the squared sound speed.

Tension.-The combined pushing of all the vortex bundles pinned onto a given magnetic flux line may globally bend the flux line. In effect, a tension force is developed that tries to resist further deformation. The average tension per unit length is given by (Harvey et al. 1986)

$$
\begin{aligned}
f_{\text {tens }} & =-\frac{9 h^{2} c^{2} \mathscr{N}_{\text {flux }}}{256 e^{2} \pi^{2} \lambda^{2}} \ln \left(\frac{\lambda}{\xi}\right) \frac{1}{s_{c}} \hat{e}_{r} \\
& \approx-\frac{R_{\text {star }} f_{\text {buoy }}}{2 s_{c}} \ln \left(\frac{\lambda}{\xi}\right) \hat{e}_{r},
\end{aligned}
$$

where $s_{c}$ is the radius of curvature of the flux tube.

Electron drag force.-As a flux tube drifts out of the core, it will experience a drag force arising from the scattering of the degenerate relativistic electron. This drag force limits the speed of the flux tubes and hence the rate of magnetic field decay. In the absence of clumping of flux tubes, the drag force per unit length is given by ${ }^{3}$ (Muslimov \& Tsygan 1985; Harvey et al. 1986; Jones 1987)

$$
f_{\text {drag }}=-\frac{27 \pi}{1024} \frac{n_{e} h^{2} c \mathscr{N}_{\text {flux }}^{3 / 2} v_{f}}{E_{f}(e) \lambda} \hat{\boldsymbol{e}}_{r},
$$

where $E_{f}(e)$ is the electron Fermi energy, $v_{f}$ is the velocity of the flux tube, and $n_{e}$ is the electron number density and is given by (Alcock et al. 1986)

$$
n_{e}=\frac{\mu_{e}^{3}}{3 \pi^{2}} .
$$

The electron chemical potential in a strange star is about 20 MeV (Benvenuto et al. 1991b), giving us $n_{e} \sim 3.5 \times 10^{34}$ $\mathrm{cm}^{-3}$. Thus, the electron number density is some 2 orders of magnitude smaller than that of a neutron star. Consequently, the electron drag force in a strange star is much weaker than that in a neutron star.

The average radial velocity of a flux tube is approximately equal to its steady state radial velocity $v_{f}$, which

\footnotetext{
${ }^{3}$ Note that there is an extra factor of $9 / 4$ because the flux quantum for proton superconductor and strange matter superconductor are different. In addition, the case of flux clumping will be addressed in $\S 4.5$.
}

can be calculated from the force balance equation

$$
f_{f, \text { Mag }}+f_{\text {buoy }}+f_{\text {tens }}+f_{\text {drag }}=0 .
$$

After finding $v_{f}$, the magnetic field $B_{\text {star }}$ of the star can be computed by solving the equation (compare with the $\dot{\Omega}$ equation in Alpar et al. 1984a)

$$
\dot{B}_{\text {star }}=-\frac{2 B_{\text {star }} v_{f}}{R_{\text {star }}}
$$

\subsection{The Alignment Torque}

In general, magnetic flux tubes incline at a nonzero angle $\theta$ to the rotational axis of the star. Thus, the magnitude (and sometimes even the direction) of force acting on a flux tube by the vortex bundles changes as we go along the flux tube itself. The combined forces acting on a magnetic flux tube due to vortex bundles, therefore, produce a total force together with a torque (Ruderman 1991a, 1991b, 1991c). While the total force may lead to a magnetic field decay in a spinning down strange star, the torque tends to push the field lines toward the equator as the star spins down. Similarly, it tends to push the field lines toward the poles as the star spins up (Ruderman 1991b).

The effect of this torque on the time evolution of magnetic field is not completely clear. If the crustal material of a strange star, which is made up of nuclear instead of strange matter, is strong enough to support a shear, then the direction of magnetic field will not change with time. On the other hand, if the crustal nuclear material is weak and brittle, the direction of the magnetic field may change via a series of "crust cracking" (Ruderman 1991a, 1991b, 1991c). A more complete discussion on the effect of this alignment torque will be reported in future works.

\subsection{The Crustal Magnetic Field}

So far, I am concentrating on the magnetic field in the core of the star. In this subsection, I show that the presence of a crustal magnetic field does not seriously affect the magnetic field evolution of a strange star. The density of the strange stellar crust must be below the neutron drip density $\left(\sim 4.3 \times 10^{11} \mathrm{~g} \mathrm{~cm}^{-3}\right)$, otherwise the dripped neutrons can convert into strange matter by making contact with them. Since the most favorable nucleus just below neutron drip is ${ }^{118} \mathrm{Kr}$ (Baym \& Pethick 1975), spacing between the nuclear lattice just below the neutron drip $b$ is about $70 \mathrm{fm}$. Hence, the Young's modulus of this lattice is about $(Z e)^{2} / b^{4} \sim 10^{29}$ dyn $\mathrm{cm}^{-2}$. (The Young's modulus of the nuclear lattice is much smaller if it is not prefect or if the density of the crust is lower than that of the neutron drip.) If the magnetic flux is completely expelled from the core, the inner layer of the normal matter crust has to sustain a stress of $\approx B^{2} \Delta R / 4 \pi$ per unit length, where $\Delta R \sim 100 \mathrm{~m}$ is the thickness of the crust. The magnetic stress lengthens the nuclear lattice; however, such a lengthening must not be greater than the $\lesssim 10 \mathrm{fm}$ electrostatic gap between strange and nuclear matter in the star. Otherwise, strange matter conversion will take place and the crust will be destroyed. After some computation, I find that the maximum field and flux the nuclear crust can sustain are $\sim 10^{7} \mathrm{G}$ and $\sim 7 \times 10^{17} \mathrm{G} \mathrm{cm}^{2}$, respectively. This is much smaller than the initial magnetic flux of a strange pulsar. So we have two possibilities: (1) there is an efficient field decay mechanism operating in the thin nuclear matter crust; or (2) the crust breaks at some 
point when majority of the field in the core is expelled, eventually making the star a bare strange matter object. In either case, the contribution of the magnetic field in the crust does not play an important role in the magnetic evolution of the entire star. Thus, we shall neglect the presence of a crustal field in our subsequent analysis.

\subsection{Magnetic Field Decay-Numerical Results}

I numerically compute the magnetic field decay due to interpinning of magnetic flux tubes and vortex bundles. I assume that the initial magnetic field $B_{\text {star }}(0)$ is uniformly distributed in the stellar core, and we also neglect the existence of a thin nuclear matter crust. The initial angular speed of the star is set to be $\Omega(0)$. The effect of the alignment torque is also ignored. To simplify calculations, we follow Ding et al. (1993) to combine the buoyancy force (eq. [39]) and the tension force terms (eq. [40]) because of their similar dependence on parameters. We write

$$
f_{\text {buoy }}+f_{\text {tens }}=\gamma f_{\text {buoy }} \text {, }
$$

where $\gamma=1-\left(R_{\text {star }} / 2 s_{c}\right) \ln (\lambda / \xi)$ is a time-dependent quantity of order of unity in most parts of the star (Harvey et al. 1986).

What happens if the magnetic flux lines form a clump? Provided that the size of such a clump is smaller than the electron mean free path, electrons may collectively scatter with a number of flux lines within a clump leading to a dramatic change in the drag force (Ruderman 1992). In addition, clumping leads to an increase in the local density of pinning centers. Moreover, the force between flux tubes within a clump may be important. Thus, our mean field estimate of the force acting on the flux tubes by the vortex bundles will change as well.

We model the clumping effect by a renormalization scheme. By coarse graining to the level of a clump, the behavior of the flux tubes inside a clump is similar to that of a single magnetic flux tube carrying the same amount of flux as the clump provided that the size of the clump is much smaller than the electron mean free path. In addition, the magnetic field strength in this single magnetic flux tube equals that in each of the individual flux tubes in the clump. That is, we may replace $\mathscr{N}_{\text {flux }}$ by $\mathscr{N}_{\text {flux }} \mathscr{N}_{\text {clump }}$. As a result, the electron drag force, vortex acting force, and the buoyancy force on a clump are given by

$$
\begin{gathered}
f_{\text {drag, coll }} \approx \mathscr{N}_{\text {clump }}^{3 / 2} f_{\text {drag }}, \\
f_{\text {Mag, coll }} \approx \mathscr{N}_{\text {clump }} f_{f, \text { Mag }},
\end{gathered}
$$

and

$$
f_{\text {buoy, coll }} \approx \mathscr{N}_{\text {clump }} \gamma f_{\text {buoy }},
$$

respectively. So the electron drag force becomes dominant when clumping of flux lines is serious or when the quark superconductivity is extremely type I. And from now on, the symbol $\mathscr{N}_{\text {flux }}$ should be interpreted as the product of the number of flux quantum in each flux tube and the number of flux tubes in a clump.

Let us consider a $1.4 M_{\odot}$ strange star with (core) radius $11 \mathrm{~km}$ whose density in the outer region of the strange core is around $5 \times 10^{14} \mathrm{~g} \mathrm{~cm}^{-3}$. The moment of inertia of the star is about $1.6 \times 10^{45} \mathrm{~g} \mathrm{~cm}^{2}$ (Benvenuto et al. 1991b). We also fix $\theta=45^{\circ}$. We take a typical star with initial magnetic field $B_{\text {star }}(0)=10^{12} \mathrm{G}$, initial angular speed $\Omega(0)=2000 \mathrm{rad}$ $\mathrm{s}^{-1}$ (and hence, an initial period of about $3 \mathrm{~ms}$ ), $\gamma=0.5$, and
$\mathscr{N}_{\text {flux }}=1$ as our "reference" star. Then the effects of the initial magnetic field, initial angular speed, the value of $\gamma$ in the effective buoyancy force, and the number of flux quanta in a flux clump $\mathscr{N}_{\text {flux }}$ on the stellar magnetic evolution can be studied by varying these parameters one at a time.

We first investigate the case when there is no clumping, and the system is almost type II. So we set $\mathscr{N}_{\text {flux }}=1$. As shown in Figures $2 a$ and $3 a$, both the magnetic field and angular speed remain approximately constant for a while, which are followed by power-law decays with exponents equal $-\frac{1}{4}$. Their transition times decrease with increasing initial field. In addition, the radial velocities of both the vortices and the fluxoids moves as a whole most of the time (see Fig. 4a).

We can explain both the field and angular speed evolution in a simple way. Since the initial magnetic field is not too high, the star only experiences a modest spin down. Thus, the vortex bundles are effectively pinned to the flux tubes (instead of thermally creeping through them) leading to what Ding et al. (1993) called a "comoving phase." In this phase, $B_{\text {star }}(t) \sim \Omega(t)$ and hence, the dipole spin down of the star is given by

$$
\dot{\Omega}=-\frac{\Omega^{3} R_{\mathrm{star}}^{6} B_{\mathrm{star}}^{2} \sin ^{2} \theta}{3 I c^{3}} \approx-\frac{B_{\mathrm{star}}^{2}(0) R_{\mathrm{star}}^{6} \Omega^{5} \sin ^{2} \theta}{2 I c^{3} \Omega^{2}(0)} .
$$

Upon integration, it is easy to show that

$$
\begin{aligned}
\Omega(t) & \approx\left[\frac{4 B_{\mathrm{star}}^{2}(0) R_{\mathrm{star}}^{6} t \sin ^{2} \theta}{3 I c^{3} \Omega^{2}(0)}+\frac{1}{\Omega^{4}(0)}\right]^{-1 / 4} \\
& \equiv\left[\frac{t}{t_{0}}+\frac{1}{\Omega^{4}(0)}\right]^{-1 / 4} .
\end{aligned}
$$

Thus, $\Omega$ (and hence, $B_{\text {star }}$ ) is almost a constant when $t \ll t_{0}$ and $\Omega$ decays as a power law with an exponent $-\frac{1}{4}$ when $t \gg t_{0}$. This is consistent with the field and angular speed evolution we have plotted in Figures $2 a$ and $3 a$.

Now, we go on to consider the case when clumping is important, and when the quark superconductor is almost type I. We illustrate the situation by putting $\mathscr{N}_{\text {flux }}=10^{6}$. As shown in Figures $2 b$ and $3 b$, when the initial field is low $\left(\lesssim 3 \times 10^{12} \mathrm{G}\right)$, the magnetic and spin evolution behave almost in same way as in the case when $\mathscr{N}_{\text {flux }}=1$. The huge value of $\mathscr{N}_{\text {flux }}$ implies that the electron drag force may be dominant. In order to push the flux tubes, the vortex bundles need to acquire a strong Magnus force by increasing the value of the angular velocity lag $\omega$. Nevertheless, $|\omega|$ cannot exceed its critical value $\omega_{\mathrm{cr}}$. So, when the star spins down too quickly, the vortex bundles in the core have no choice but to thermally creep through the flux tubes leading to what Ding et al. (1993) called the "forward creeping phase." This picture is consistent with the numerical finding that forward creeping phase lengthens with increasing initial magnetic field (see Figs. $4 b, 4 c$, and $4 d$ ). The small radial velocity of the flux tubes at this phase implies that $B_{\text {star }}$ remains almost constant. It is until the onset of the comoving phase due to a much slower spin-down rate at later times that $B_{\text {star }}$ and $\Omega$ decay like $t^{-1 / 4}$ (see Figs. $2 b$ and $3 b$ ).

Now we discuss a more interesting case when $B_{\text {star }}(0)=$ $10^{13} \mathrm{G}$ and $\mathscr{N}_{\text {flux }}=10^{6}$. Figure $2 b$ shows that $B_{\text {star }}$ decays exponentially with a characteristic time of about $5 \mathrm{Myr}$. However, at about $100 \mathrm{Myr}$, the decay stays almost constant for a while, and then it decays further like $t^{-1 / 3}$. 


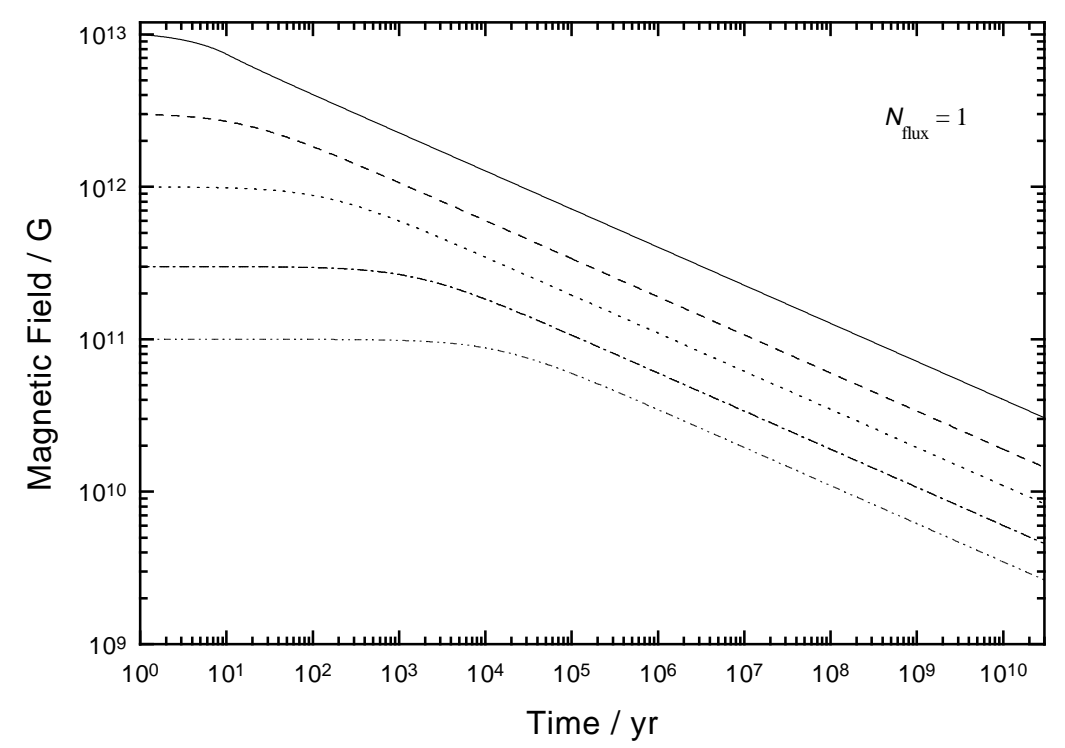

FIG. $2 a$

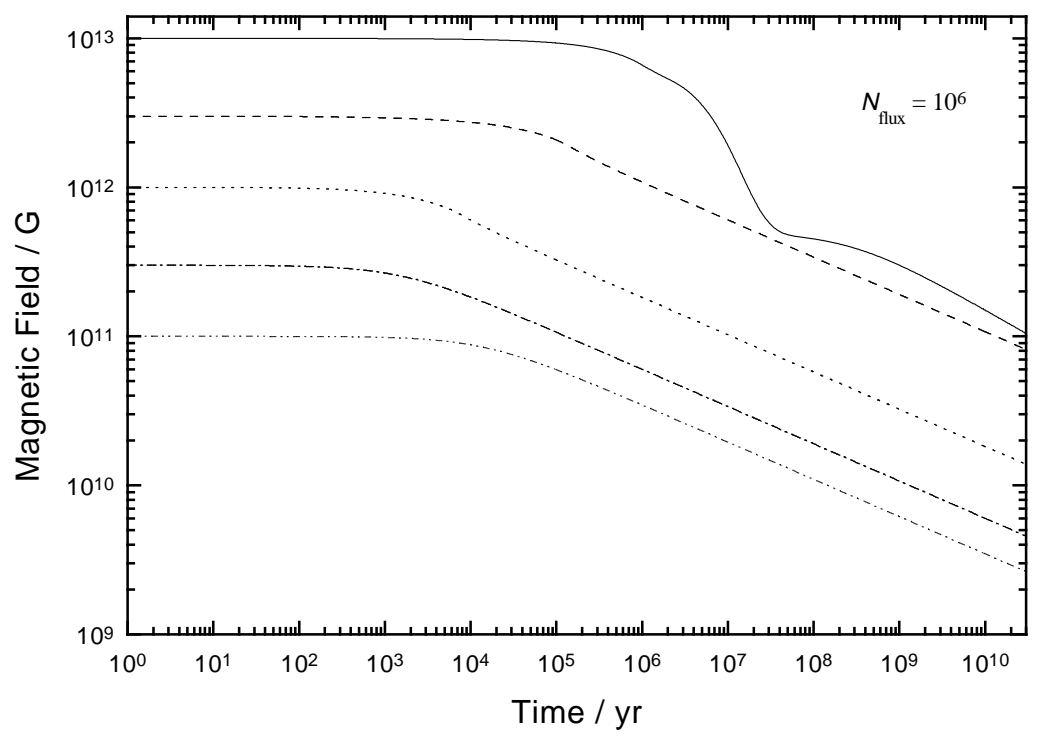

FIG. $2 b$

FIG. 2.-Magnetic field decay of a strange star as a function of its initial $B$ field. The initial field measured in $\mathrm{G}$ is varied from $10^{13}$ (solid line), $3 \times 10^{12}$ (dashed line), $10^{12}$ (dotted line), $3 \times 10^{11}$ (dash-dotted line), to $10^{11}$ (short dash-dotted line). Panel $(a)$ shows the field decay when $\mathscr{N}_{\text {flux }}=1$, while panel (b) shows the field decay when $\mathscr{N}_{\text {flux }}=10^{6}$.

Besides, Figure $3 b$ shows that $\Omega$ decreases like a power law with an exponent $-\frac{1}{2}$ for almost $10 \mathrm{Myr}$. After that, $\Omega$ stays almost constant for a while but this is then followed by a power-law decay with an exponent about $-\frac{1}{6}$.

We can explain the behavior as follows: The rapid spin down due to high value of $B_{\text {star }}$ leads to the forward creeping phase (see Fig. 4d). So

$$
\dot{\Omega} \approx-\frac{\Omega^{3} R_{\mathrm{star}}^{6} B_{\mathrm{star}}^{2}(0) \sin ^{2} \theta}{3 I c^{3}},
$$

and hence,

$$
\Omega(t) \approx\left[\frac{2 B_{\mathrm{star}}^{2}(0) R_{\mathrm{star}}^{6} t \sin ^{2} \theta}{3 I c^{3}}+\frac{1}{\Omega^{2}(0)}\right]^{-1 / 2} .
$$

This accounts for the exponential decay in $\Omega$ starting from $3 I c^{3} / 2 B_{\text {star }}^{2}(0) R_{\text {star }}^{6} \Omega^{2}(0) \sin ^{2} \theta \approx 5$ yr. About $10^{3}$ yr or so, the star rotates so slowly that vortex bundle density becomes very low. At this moment, buoyancy becomes the dominant driving force for field decay and hence,

$$
\dot{B}_{\text {star }}=-\frac{2 B_{\text {star }} v_{f}}{R_{\text {star }}} \approx-\frac{16 \gamma E_{f}(e) c B_{\text {star }}}{3 \pi^{3} e^{2} n_{e} \lambda R_{\text {star }}^{2} \mathscr{N}_{\text {flux }}^{1 / 2}} \equiv-\frac{B_{\text {star }}}{\tau_{\text {buoy }}} .
$$

That is, $B_{\text {star }}$ decays exponentially with a characteristic time $\tau_{\text {buoy }}$ of about 4 Myr. Finally, at time $\gtrsim 10 \mathrm{Myr}$, dipole spin down of the star becomes very ineffective due to the small values of $\Omega$ and $B_{\text {star }}$. At this time the radial velocity of flux tubes is faster than that of the vortex bundles and the star enters the "reverse creeping" phase (see Fig. $4 d$ and Ding et al. 1993). The pinning force prevents the flux tubes from moving too fast, and hence, the rate of field decay is decreased. The force balance equation for the flux tubes 


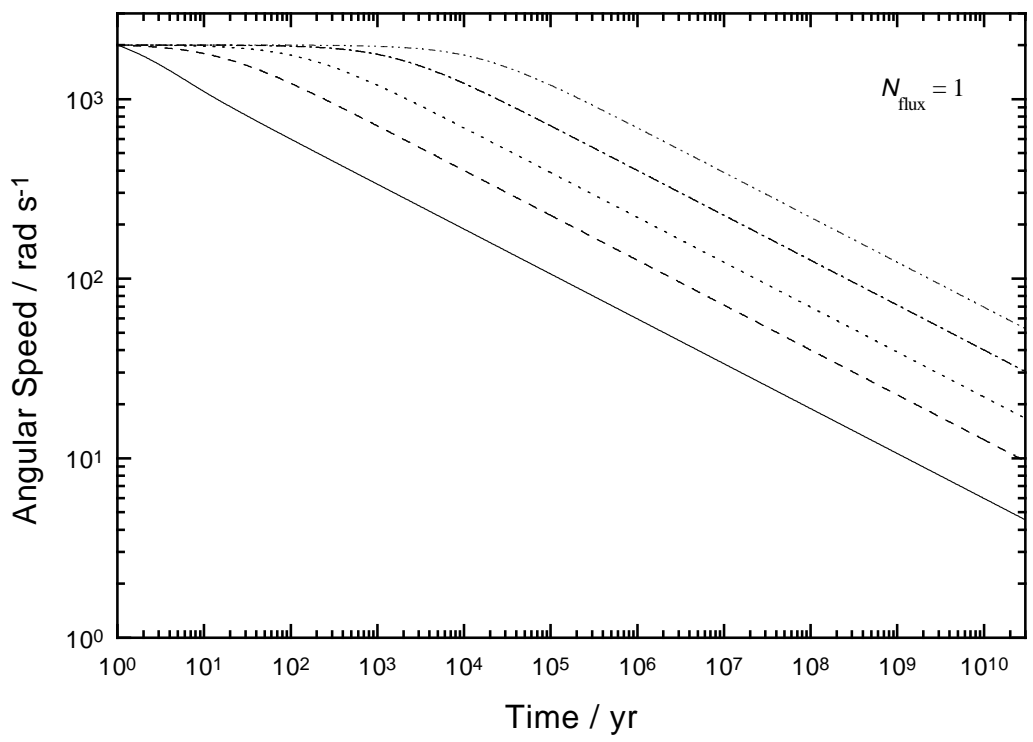

FIG. $3 a$

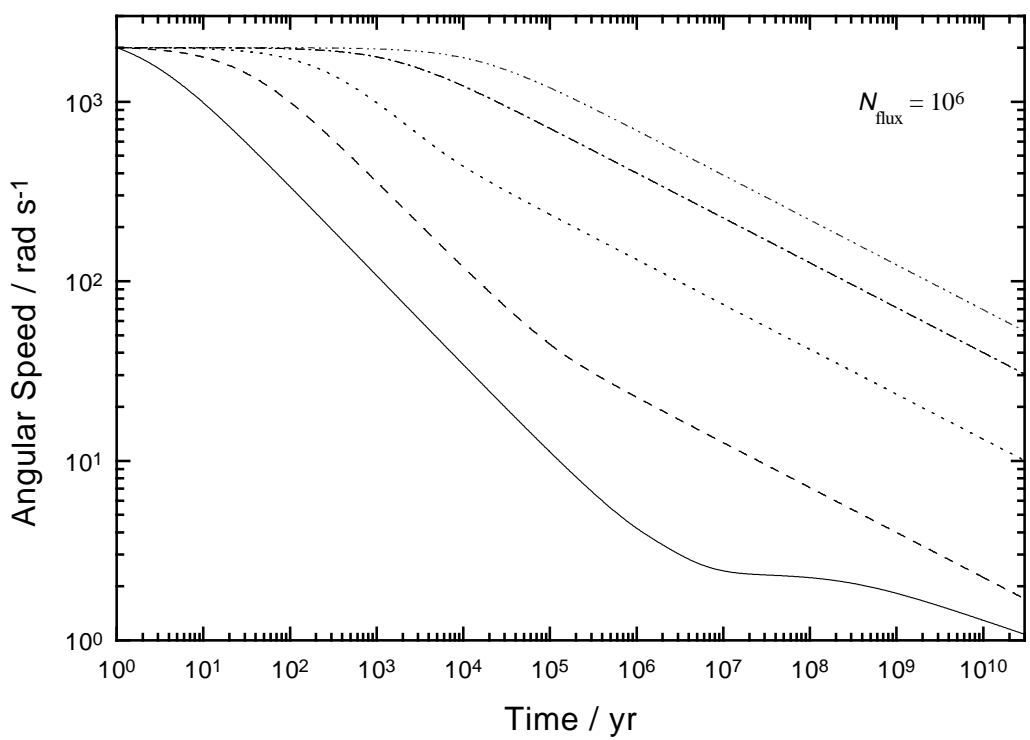

FIG. $3 b$

Fig. 3.-Spin down of a strange pulsar as a function of its initial magnetic field. The solid, dotted, dashed, dash-dotted, and short dash-dotted lines correspond to initial magnetic fields of $10^{11}, 3 \times 10^{11}, 10^{12}, 3 \times 10^{12}$, and $10^{13} \mathrm{G}$, respectively. Panels $(a)$ and $(b)$ are for $\mathcal{N}_{\text {flux }}=1$ and $10^{6}$, respectively.

reads $f_{\text {buoy, coll }}-f_{\text {Mag, coll }}=f_{\text {drag, coll }} \approx 0$ (compare with eq. [43]). Since $f_{\text {buoy,coll }}$ is time independent, $f_{\text {Mag, coll }} \propto$ $\left|\Omega \omega / B_{\text {star }}\right|$, and $\omega \approx-\omega_{\text {cr }} \propto\left|B_{\text {star }}\right|^{1 / 2}$, the force balance equation implies that $B_{\text {star }} \sim \Omega^{2}$. As a result, $\Omega(t) \sim t^{-1 / 6}$ and $B_{\text {star }}(t) \sim t^{-1 / 3}$ at the very late stage of the evolution of the star (see Figs. $2 b$ and $3 b$ ).

We now turn to the study of the effect of $\Omega(0)$ to the magnetic evolution of the star. Figure 5 shows that the field decays more rapidly (due to the faster onset of the powerlaw decay) as $\Omega(0)$ increases when the star is in comoving phase. This agrees with the crossover time estimate given in equation (48). However, if the star is in the forward creeping phase, the push received by the flux tubes due to pinned vortices saturates (and thus, becomes independent of $\Omega$ ). So in this case, the value of $\Omega(0)$ has little effect on the magnetic evolution of the star. I have verified this in some of our runs.

Next, we consider the effect of $\gamma$. Again, the only situation in which the value of $\gamma$ can seriously affect the magnetic evolution of a star is when the pinning force is weak at some moment as compared to the buoyancy force; and this happens when both $B_{\text {star }}(0)$ and $\mathscr{N}_{\text {flux }}$ are large. Figure 6 show the magnetic field evolution when $B_{\text {star }}(0)=10^{13} \mathrm{G}$ and $\mathscr{N}_{\text {flux }}=10^{6}$. We see that the evolution profile depends quite sensitively on $\gamma$. When $\gamma=0$, we observe the $B_{\text {star }} \sim$ $t^{-1 / 4}$ behavior at late times, indicating that the star is in the comoving phase. This is reasonable, since the only force that push the flux tubes out from the core comes from the vortex bundles. But when $\gamma \gtrsim 0.5$, the buoyancy force dominates. Thus, the magnetic evolution follows an exponential decay when $t \lesssim 10^{6} \mathrm{yr}$ and $B_{\text {star }} \sim t^{-1 / 3}$ at $t \gtrsim 10^{9} \mathrm{yr}$, indicating the presence of forward creeping and reverse creeping phase at early and late times, respectively.

Finally, we consider the effect of $\mathscr{N}_{\text {flux }}$. For $B_{\text {star }}(0)=$ $10^{12} \mathrm{G}$, Figure $7 a$ tells us that the behavior of the star does not have any visible change $\mathscr{N}_{\text {flux }}$ increases from 1 to $10^{3}$. They are all in the comoving phase. This observation is 


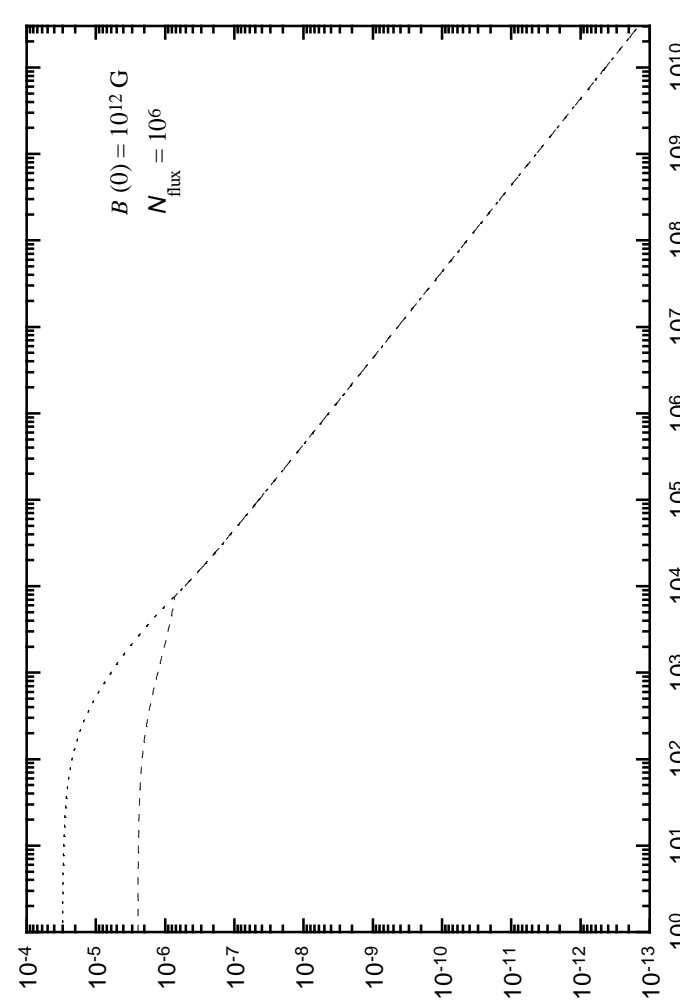

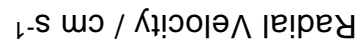

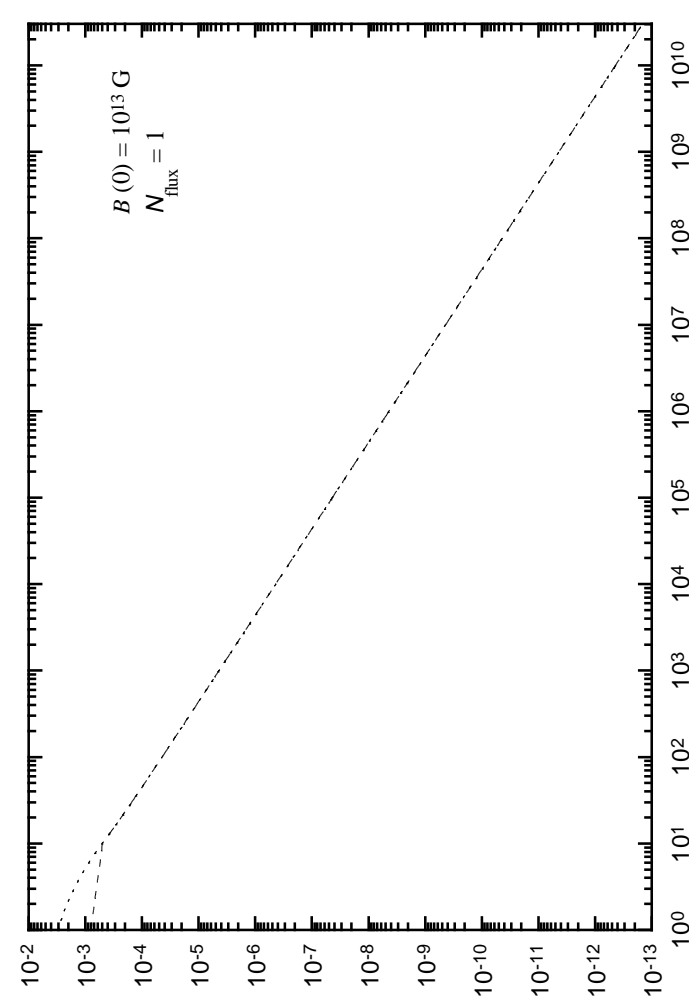

ı-s mo / Кıрооә^ ןе!pey

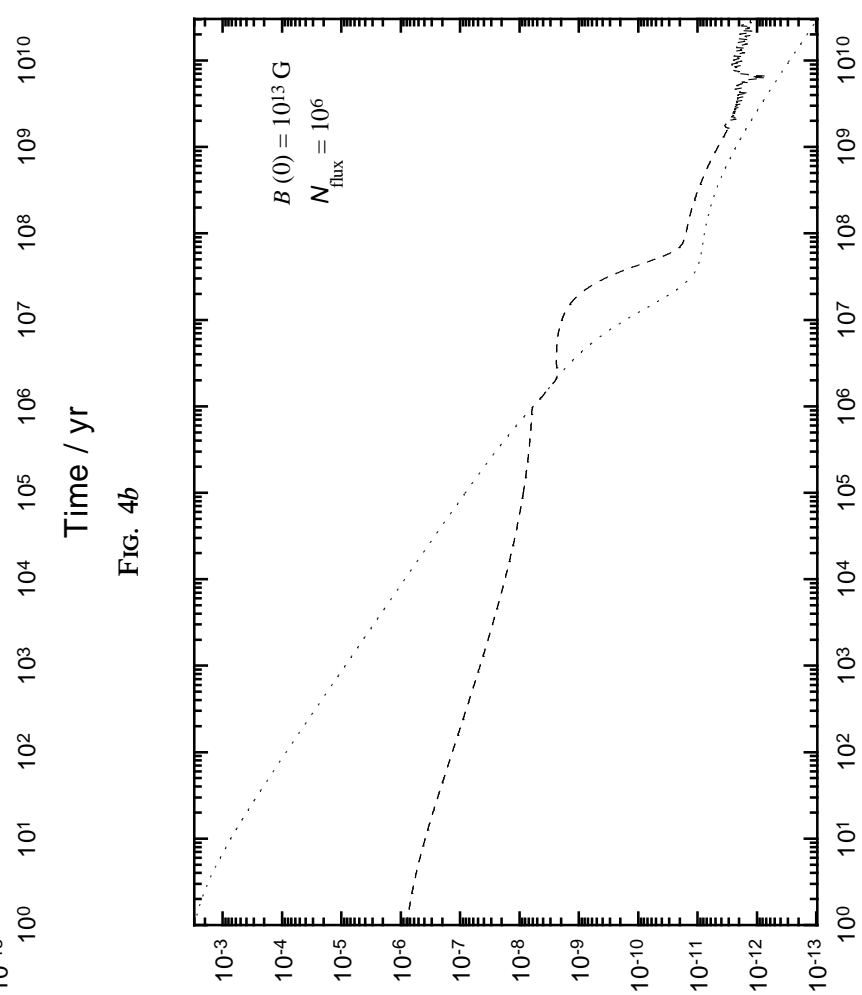

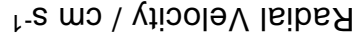

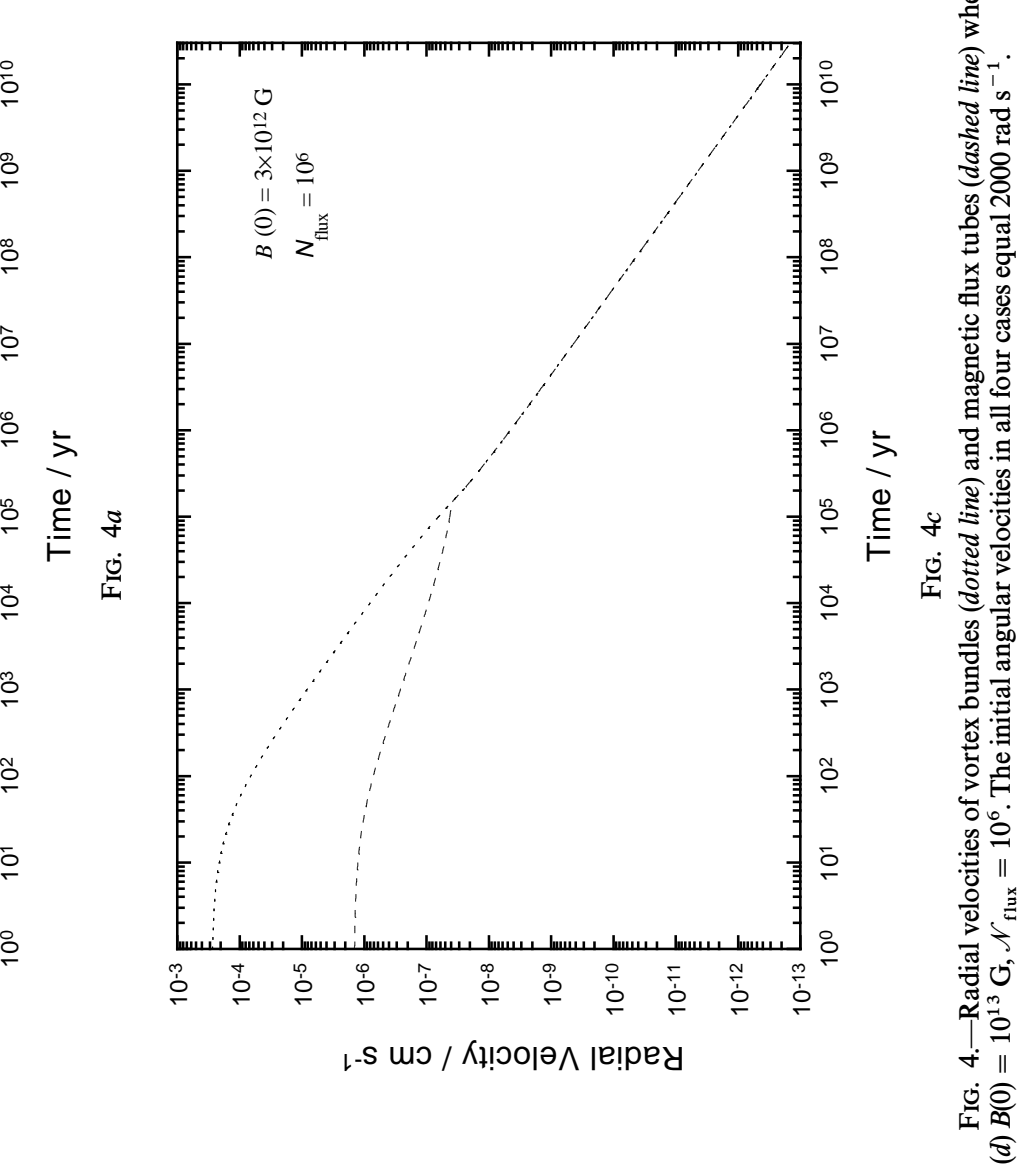




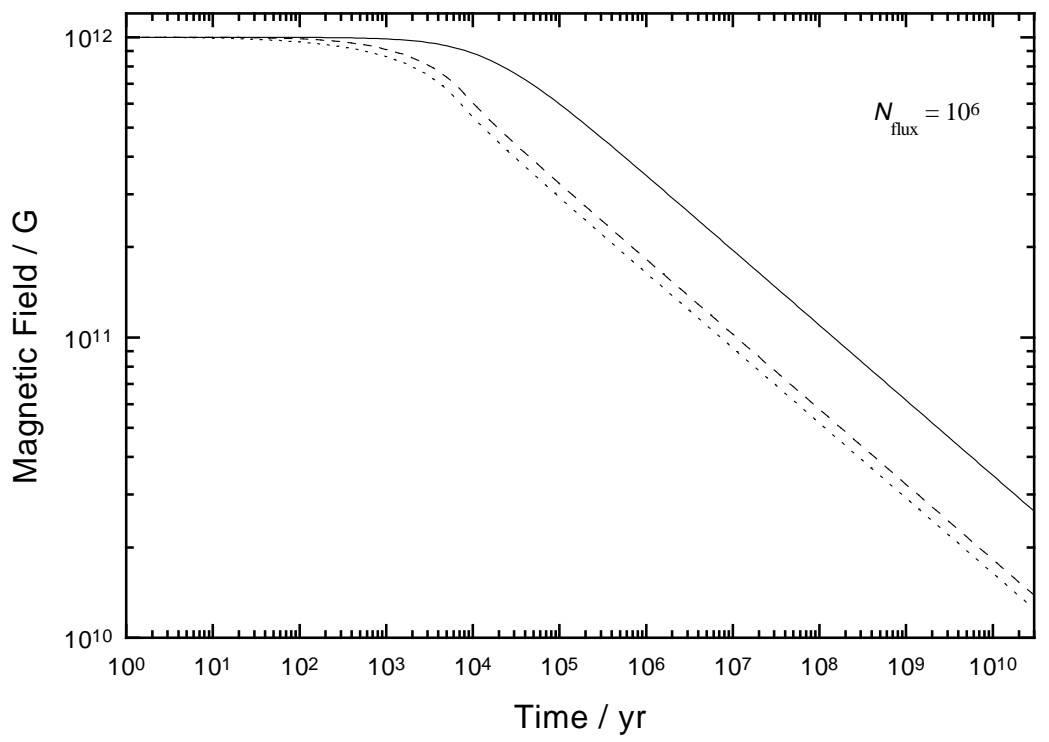

FIG. 5.-Magnetic field evolution as a function of initial angular speed. The solid, dashed, and dotted lines correspond to initial angular speed of 200, 2000 , and $12,000 \mathrm{rad} \mathrm{s}^{-1}$, respectively. $\mathscr{N}_{\text {flux }}$ is set to $10^{6}$.

consistent with the fact that $t_{0}$ in equation (48) is independent of $\mathscr{N}_{\text {flux }}$. It is only when $\mathscr{N}_{\text {flux }}$ increases to about $10^{6}$ we begin to see a slow down of field decay due to the presence of a relatively long period of forward creeping phase before the star eventually enters the comoving phase.

The magnetic evolution is more dramatic if we take $10^{13}$ $\mathrm{G}$ as the initial field. Figure $7 b$ shows that when $\mathscr{N}_{\text {flux }}=1$, the star is basically in the comoving phase all the time. When $\mathscr{N}_{\text {flux }}=10^{3}$, a delay in field decay is observed due to the presence of a long period of forward creeping phase at early times. Finally, when $\mathscr{N}_{\text {flux }}=10^{6}$, the star switches to an initial exponential, followed by an eventual power-law delay mode, indicating that the star has locked into the forward creeping $\rightarrow$ comoving $\rightarrow$ reverse creeping pattern.

In summary, we find that in all reasonable parameter ranges, the value of the initial magnetic field will be reduced to $1 / e$ of its original value in less than $1 \mathrm{Myr}$ time when
$\mathscr{N}_{\text {flux }} \sim 1$ or when $B_{\text {star }}(0) \lesssim 10^{12}$ G. If $\mathscr{N}_{\text {flux }} \sim 10^{6}$, the characteristic field decay time is less than $20 \mathrm{Myr}$ provided that $B_{\text {star }}(0) \lesssim 3 \times 10^{12} \mathrm{G}$ or $\gamma \sim 0$. The only way we obtain a characteristic decay time longer than $20 \mathrm{Myr}$ is by using $\mathscr{N}_{\text {flux }}$ as high as $10^{6}, B_{\text {star }}(0)$ as high as $10^{13} \mathrm{G}$, and $\gamma \sim 0$ (see Fig. 6). This combination of parameters is unlikely to occur physically.

\subsection{Observational Implications of Strange Star Field Decay}

The presence of a large number of systematic and random errors in the observed pulsar sample together with the fact that kinematic age sometimes does not truly reflect the real age of a pulsar greatly complicate the analysis of pulsar magnetic field decay. Nonetheless, current statistical analysis and computer simulation of Galactic pulsar distribution suggest that the characteristic field decay time is at least 20 Myr (Bhattacharya et al. 1992; Wijers et al. 1993;

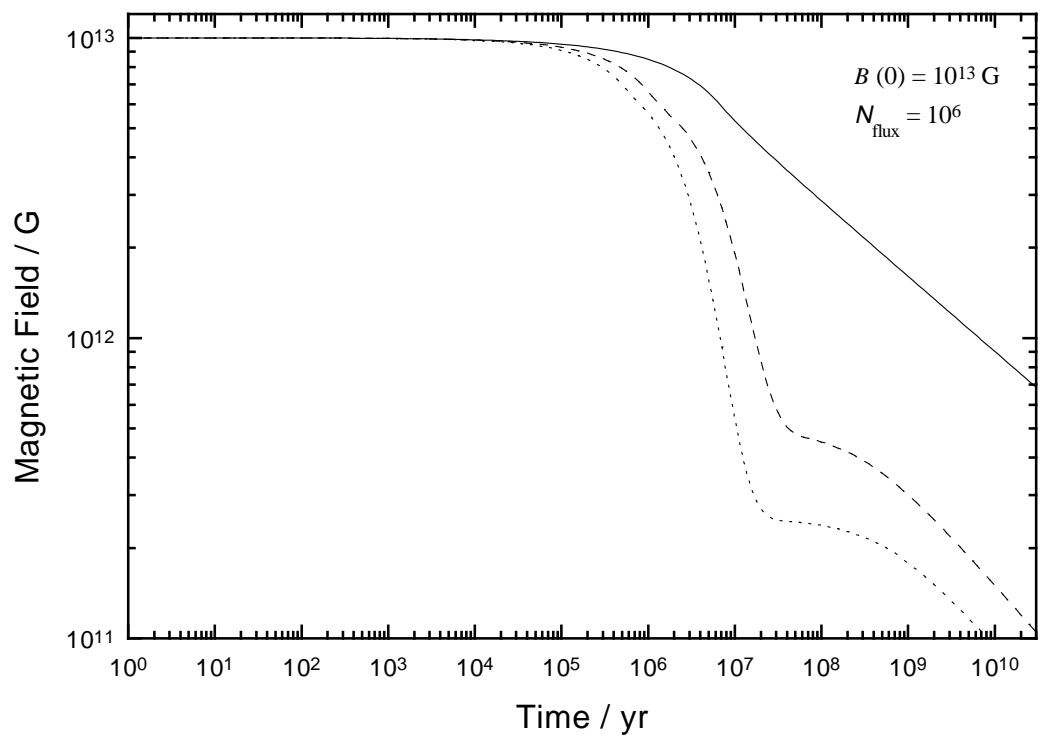

FIG. 6.-Magnetic field evolution as a function of the value $\gamma$. The solid, dotted, and dashed lines correspond to $\gamma=0,0.5$, and 1 , respectively. Note that the initial magnetic field is set to $10^{13} \mathrm{G}$. 


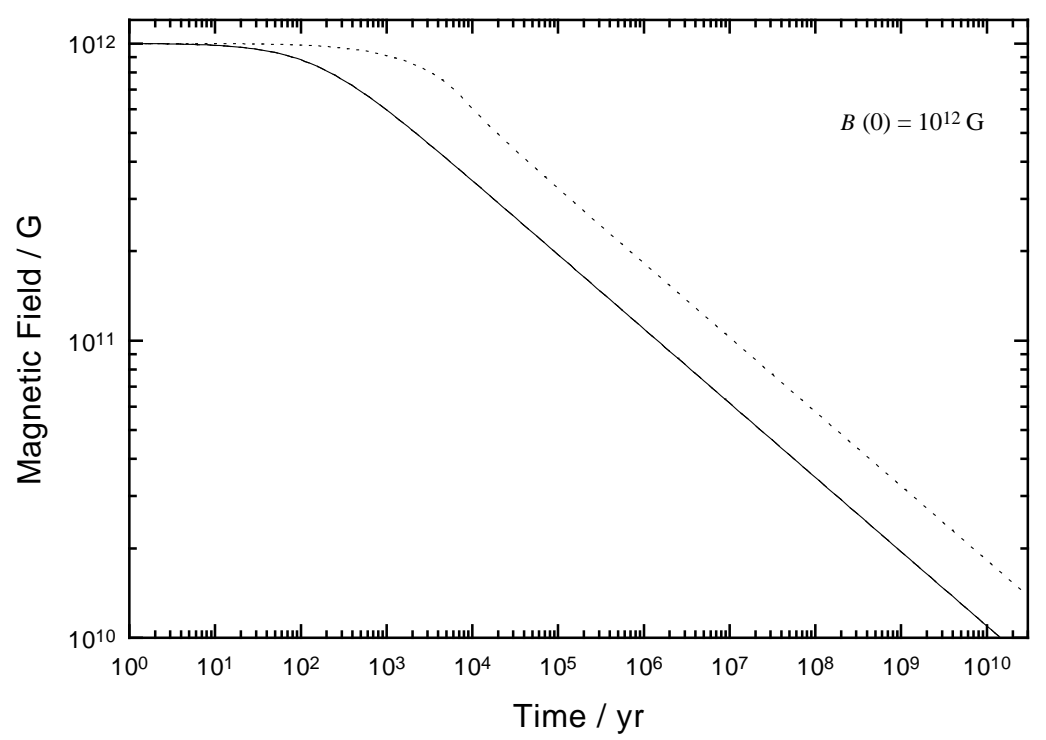

FIG. $7 a$

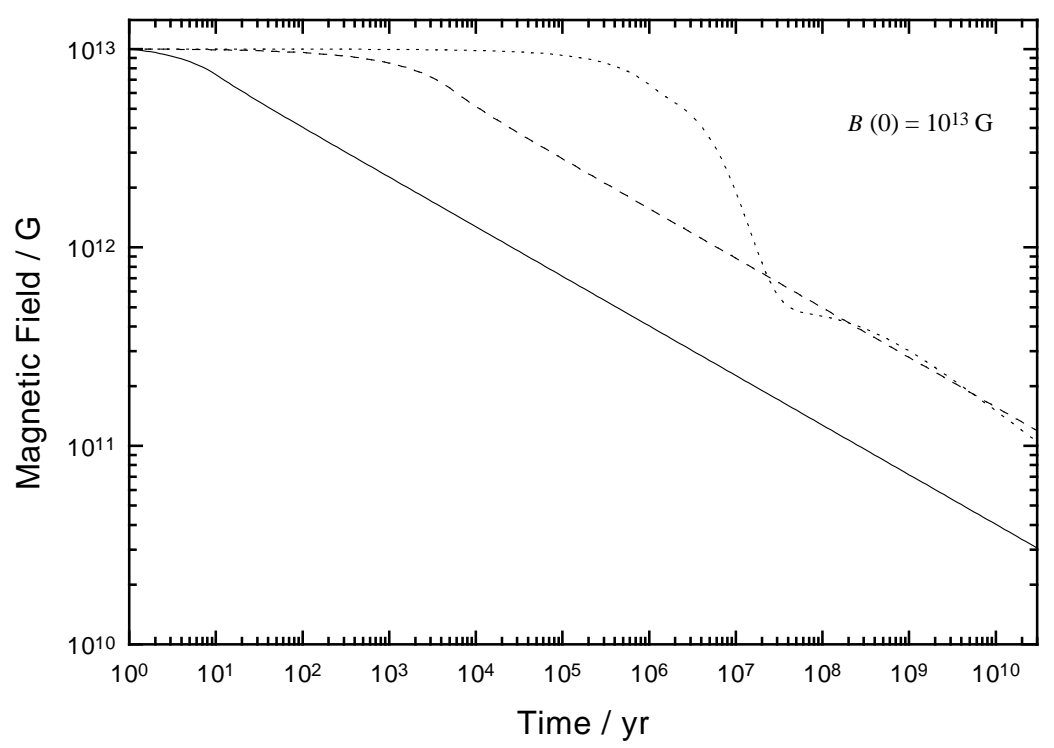

FIG. $7 b$

FIG. 7.- Magnetic field evolution as a function of $\mathscr{N}_{\text {flux }}$. The solid, dashed, and dotted lines correspond to $\mathscr{N}_{\text {flux }}=1,10^{3}$ and $10^{6}$, respectively. Panel $(a)$ shows the field decay when $B(0)=10^{12} \mathrm{G}$, and panel $(b)$ shows the field decay when $B(0)=10^{13} \mathrm{G}$. Note that the solid curve overlaps with the dashed one in $(a)$.

see also the recent review by Bhattacharya \& Srinivasan 1995).

Various authors have studied theoretically the possibility of a magnetic field decay in the core of a neutron star (Sauls 1989; Srinivasan et al. 1990; Chau et al. 1992; Ding et al. 1993). However, Pethick \& Sahrling (1995) pointed out recently that the field decay time in the inner crust of a neutron star is at least $80 \mathrm{Myr}$ due to the slow diffusion of the field through the inner crust. Consequently, core magnetic field decay only results in transporting the field from the stellar core to the inner crust; the total stellar field remains unchanged in $\gtrsim 80 \mathrm{Myr}$. Therefore, the conventional hypothesis that pulsars are neutron stars is consistent with the observed field decay time of the star.

On the other hand, as suggested by our numerical finding in $\S 4.5$, the magnetic field of a strange star decays with a characteristic times $\leq 20 \mathrm{Myr}$. In fact, equation (51) tells us that the characteristic time for the buoyancy force dominated field decay $\tau_{\text {buoy }}$ equals $4.2 \times 10^{-3} \mathscr{N}_{\text {flux }}$ Myr. Thus, $\tau_{\text {buoy }}$ is longer than $20 \mathrm{Myr}$ only when $\mathscr{N}_{\text {flux }} \geq 2 \times 10^{7}$-a value that is attainable only when the quark superconductor is extremely type I. Therefore, our finding is inconsistent with the proposition that pulsars are strange stars.

\section{CONCLUSIONS}

In summary, I consider the rotation of a multisuperconducting species object. The rotation of such an object is made possible by the formation of vortices similar to that of superfluid helium. This finding implies the existence of vortex bundles in the core of a rotating superconducting strange star. Because it is energetically favorable for the vortex bundles to pin to magnetic flux tubes, the rotational dynamics and the magnetic field evolution of a strange star are coupled. 
Because the nuclear crust of a strange star cannot sustain a strong magnetic stress, the magnetic field evolution of the star is dictated by dynamics of the flux tubes in the core. The core magnetic field evolution due to interpinning of magnetic flux tubes and vortex bundles, and clumping of flux tubes is computed numerically. I find that in all reasonable parameter ranges, the characteristic decay time of the magnetic field is $\leq 20 \mathrm{Myr}$. This finding does not agree with the hypothesis that pulsars are superconducting strange stars because current pulsar data strongly suggest that pulsar magnetic fields do not decay in $20 \mathrm{Myr}$.

A number of interesting questions remains. The phase diagram of interacting multiple component superconducting species in rotation remains unclear. It is also interesting to study the effects of alignment torque on magnetic and rotational history of a strange star, and the possible collapse of the nuclear matter crust due to magnetic stress. I plan to report them in my future works.

I would like to thank Mal Ruderman for bringing up this problem to my attention and K. Y. Ding for providing us her field decay program for neutron stars for reference. I would also like to thank H.-K. Lo, Geoff Ravenhall and Frank Wilczek for their useful discussions, and Mark Alford for critically read through this manuscript. The hospitality of Aspen Center for Physics is acknowledged where the early part of this work was performed in the summer of 1995. This work is supported by DOE grant DE-FG0290ER40542.

\section{APPENDIX A}

\section{SOLUTION TO THE STRONGLY INTERACTING SUPERCONDUCTING SPECIES PROBLEM}

We prove the following claim by explicitly constructing a solution to the problem. For simplicity, we shall drop all the star superscripts over the masses and charges.

Claim: Suppose there is a finite number of superconducting species. Then equation (21) has a solution with $K \neq 0$ (and hence, it has infinitely many solutions) if and only if there exist two vectors $v_{1} \equiv\left(M_{1}, Q_{1}\right)$ and $v_{2} \equiv\left(M_{2}, Q_{2}\right)$ among the $\left(m_{j}, q_{j}\right)$ together with two rational numbers $\alpha_{1}, \alpha_{2}$ such that $\left(m_{j}, q_{j}\right)=\beta_{j 1} v_{1}+\beta_{j 2} v_{2}$, with $\left\{1, \beta_{j 1}, \beta_{j 2}\right\}$ being linearly dependent over the set of all rational numbers $Q$ for all $j$. In addition, $\alpha_{1} \beta_{j 1}+\alpha_{2} \beta_{j 2}$ are rational numbers for all $j$, and $\alpha_{1} Q_{2} \neq$ $\alpha_{2} Q_{1}$.

Proof. By replacing vectors $v_{i}$ by $v_{i} / \ell(i=1,2)$, where $\ell$ is the least common multiple of the denominators of $\alpha_{1} \beta_{j 1}+\alpha_{2} \beta_{j 2}$, then $\beta_{j i}$ will be replaced by $\ell \beta_{j i}$. In addition, we can replace $\alpha_{i}$ by $\lambda \alpha_{i}$, where $\lambda$ is the least common multiple of the denominators of $\alpha_{i}$ and $\alpha_{1} \beta_{j 1}+\alpha_{2} \beta_{j 2}$. Then it is easy to verify that $\lambda \alpha_{i}$ and $\ell\left(\alpha_{1} \beta_{j 1}+\alpha_{2} \beta_{j 2}\right)$ are all integers. Thus, we can always assume that $\alpha_{1}, \alpha_{2}$, and $\alpha_{1} \beta_{j 1}+\alpha_{2} \beta_{j 2}$ to be integers instead of rational numbers.

Suppose $v_{1}$ and $v_{2}$ are linearly dependent on each other; then clearly the ratio of $m_{j}$ to $q_{j}$ are the same for all $j$. So, by choosing $N_{j}=0$ for all $j, K=1$, and $\Phi_{v}=m_{j} c / q_{j}$, equation (21) is satisfied. Now, we consider the more interesting case when $v_{1}$ and $v_{2}$ are linearly independent vectors. Consider the equation

$$
\left(\begin{array}{l}
P_{1} \\
P_{2}
\end{array}\right)=\left(\begin{array}{ll}
M_{1} & -Q_{1} \\
M_{2} & -Q_{2}
\end{array}\right)\left(\begin{array}{c}
K / h \\
\Phi_{v} / h c
\end{array}\right)
$$

The linear independence of $v_{1}$ and $v_{2}$ implies that $M_{1} Q_{2}-M_{2} Q_{1} \neq 0$, and the solution of the above equation is given by

$$
\left(\begin{array}{c}
K / h \\
\Phi_{v} / h c
\end{array}\right)=\frac{1}{M_{2} Q_{1}-M_{1} Q_{2}}\left(\begin{array}{c}
Q_{1} P_{2}-Q_{2} P_{1} \\
M_{1} P_{2}-M_{2} P_{1}
\end{array}\right)
$$

Clearly, by choosing $P_{1}=\alpha_{1}$ and $P_{2}=\alpha_{2}$, we can check that the $K$ given by equation (A2) is nonzero.

Now for each $j$, it is easy to check that by choosing $N_{j}=\alpha_{1} \beta_{j 1}+\alpha_{2} \beta_{j 2}$ (which is an integer, and this is possible because $\left\{1, \beta_{j 1}, \beta_{j 2}\right\}$ is linearly dependent over $\left.Q\right)$ together with $K(\neq 0), \Phi_{v}$ given in equation (A2), then equation (21) is satisfied. (Clearly, if $N_{j}, K, \Phi_{v}$ is a solution of eq. [21], then so is $\lambda N_{j}, \lambda K, \lambda \Phi_{v}$ for any nonzero integer $\lambda$. Thus, eq. [21] has either no solution, or infinitely many solutions.)

On the contrary, suppose that for any linearly independent vectors $v_{1}$ and $v_{2}$ chosen among $\left(m_{j}, q_{j}\right)$, and any rational numbers $\alpha_{1}$ and $\alpha_{2}$, we can find an $j$ such that $\left(m_{j}, q_{j}\right)=\beta_{j 1} v_{1}+\beta_{j 2} v_{2}$ with either (1) $\left\{1, \beta_{j 1}, \beta_{j 2}\right\}$ being linearly independent over $Q$; or (2) $\alpha_{1} \beta_{j 1}+\alpha_{2} \beta_{j 2}$ is irrational; or (3) $\alpha_{1} Q_{2}=\alpha_{2} Q_{1}$. Now we analyze these three cases one by one:

Case (1).-If $\left\{1, \beta_{j 1}, \beta_{j 2}\right\}$ is linearly independent over $Q$, then from equation (A1), we know that $N_{j}=P_{1} \beta_{j 1}+P_{2} \beta_{j 2}$. In order for $P_{1}, P_{2}$ and $N_{j}$ to be integers, the only possibility is $P_{1}=P_{2}=N_{j}=0$. However, from equation (A2), this implies $K=0$, and hence, equation (21) has no solution for $K \neq 0$.

Case (2).- If $\alpha_{1} \beta_{j 1}+\alpha_{2} \beta_{j 2}$ is irrational, then, similar to the argument in case (1), $N_{j}=\alpha_{1} \beta_{j 1}+\alpha_{2} \beta_{j 2}$ is not an integer. Hence, this choice of $\alpha_{1}$ and $\alpha_{2}$ does not produce a solution for equation (21).

Case (3).-If $\alpha_{1} Q_{2}=\alpha_{2} Q_{1}$, then $K=0$ from equation (A2), which is impossible.

The above claim implies that there is, in general, no solution to the strongly interacting limit when the number of different species of superconducting Cooper pairs is $\geq 3$. On the other hand, the solution of equation (21) always exists when there are only two species of Cooper pairs, as in the case of strange matter. 


\section{REFERENCES}

Alcock, C. 1991, Nucl. Phys. B, 24, 93

Alcock, C., Farhi, E., \& Olinto, A. 1986, ApJ, 310, 261

Alpar, M. A. 1987, Phys. Rev. Lett., 58, 2152

Alpar, M. A., Anderson, P. W., Pines, D., \& Shaham, J. 1984a, ApJ, 276, 325

Alpar, M. A., Chau, H. F., Cheng, K. S., \& Pines, D. 1993, ApJ, 409, 345

Alpar, M. A., Langer, S. A., \& Sauls, J. 1984b, ApJ, 282, 533

Andreev, A. F., \& Bashkin, E. P. 1976, Soviet Phys. JETP, 42, 164

Bailin, B., \& Love, A. 1982, Nucl. Phys. B, 205, 119 .1984, Phys. Rep., 107, 325

Baym, G. 1988, in Frontiers And Borderlines in Many-Particle Physics, ed. R. A. Broglia \& J. R. Schrieffer (Bologna: Italian Phys. Soc.), 330

Baym, G., \& Pethick, C. J. 1975, Ann. Rev. Nucl. Sci., 25, 27

Baym, G., Pethick, C. J., \& Pines, D. 1969, Nature, 224, 673

Benvenuto, O. G., \& Horvath, J. E. 1989, MNRAS, 241, 43

Benvenuto, O. G., Horvath, J. E., \& Vucetich, H. 1991a, Phys. Rev. D, 44, 1321

1991b, Int. J. Mod. Phys. A, 6, 4769

Benvenuto, O. G., \& Vucetich, H. 1991, Nucl. Phys. B, 24, 160

Benvenuto, O. G., Vucetich, H., \& Horvath, J. E. 1994, MNRAS, 266, 690

Bhattacharya, D., \& Srinivasan, G. 1995, in X-Ray Binaries, ed. W. H. G.

Lewin, J. van Paradijs, \& E. P. J. van den Heuvel (New York: CUP), 495

Bhattacharya, D., \& Van den Heuvel, E. P. J. 1991, Phys. Rep., 203, 1

Bhattacharya, D., Wijers, R. A. M. J., Hartman, J. W., \& Verbunt, F. 1992, A\&A, 254, 198

Cabrera, B. 1987, Japanese J. Appl. Phys. (Supp.), 26, 1961

Chau, H. F., Cheng, K. S., \& Ding, K. Y. 1992, ApJ, 399, 213

Ding, K. Y., Cheng, K. S., \& Chau, H. F. 1993, ApJ, 408, 167

Farhi, E., \& Jaffe, R. L. 1984, Phys. Rev. D, 30, 2379

Haensel, P., Zdunik, J. L., \& Schaeffer, R. 1986, A\&A, 160, 121

Harvey, J. A., Ruderman, M. A., \& Shaham, J. 1986, Phys. Rev. D, 33, 2084
Jones, P. B. 1986, MNRAS, 222, 577 1987, MNRAS, 228, 513 . 1988, MNRAS, 233, 875

Khalatnikov, I. M. 1957, Soviet Phys. JETP, 5, 542

Leggett, A. J. 1991, in Low Temperature Physics, ed. M. J. R. Hoch \& R. H. Lemmer (New York: Springer), 1

Lewin, W. H. G., Van Paradijs, J., \& Taam, R. E. 1991, Space Sci. Rev., 62, 223

Link, B., Epstein, R. I., \& Van Riper, K. A. 1992, Nature, 359, 616

Madsen, J., \& Haensel, P., eds. 1991, Nucl. Phys. B, Vol 24

Mendell, G., \& Lindblom, L. 1991, Ann. Phys., 205, 110

Muslimov, A. G., \& Tsygan, A. I. 1985, Ap\&SS, 115, 41

Page, D. 1992, in Proc. Workshop on High Energy Phenomenology, ed. M. A. Pérez \& R. Huerta (Singapore: World Scientific), 347

Pethick, C. J., \& Sahrling, M. 1995, ApJ, 453, L29

Ruderman, M. 1991a, ApJ, 366, 261

.1991b, ApJ, 382, 576 1991c, ApJ, 382, 587

1992, private communication

Sauls, J. A. 1989, in Timing Neutron Stars, ed. H. Ögelman \& E. P. J. van den Heuvel (Dordrecht: Kluwer), 457

Srinivasan, G., Bhattacharya, D., Muslimov, A. G., \& Tsygan, A. I. 1990, Current Sci., 59, 31

Vardanyan, G. A., \& Sedrakyan, D. M. 1981, Soviet Phys. JETP, 54, 919

Vollhardt, D., \& Wölfle, P. 1990, The Superfluid Phases Of Helium 3 (London: Taylor \& Francis)

Wijers, R. A. M. J., Verbunt, F., Bhattacharya, D., \& Hartman, J. W. 1993, in Isolated Pulsars, ed. K. A. van Riper, R. Epstein, \& C. Ho (New York: CUP), 83

Witten, E. 1984, Phys. Rev. D, 30, 272 